\begin{tabular}{|c|l|}
\hline Title & $\begin{array}{l}\text { A relaxation-accelerated propagator method for calculations of electron energy distribution function and electron } \\
\text { transport parameters in gas under dc electric fields }\end{array}$ \\
\hline Author(s) & Sugawara, Hirotake \\
\hline Citation & $\begin{array}{l}\text { Plasma Sources Science and Technology, 26(4), 044002 } \\
\text { https://doi.org/10.1088/1361-6595/aa5d7f }\end{array}$ \\
\hline Issue Date & 2017-0401 \\
\hline Doc URL & http://hdl.handle.net/2115/68648 \\
\hline Rights & Copyright @2017 IOP Publishing Ltd. \\
\hline Type & article (author version) \\
\hline File Information & Sugawara 2017-PSST-HUSCA P.pdf \\
\hline
\end{tabular}

Instructions for use 


\title{
A relaxation-accelerated propagator method for calculations of electron energy distribution function and electron transport parameters in gas under dc electric fields $\ddagger$
}

\author{
Hirotake Sugawara \\ Division of Electronics for Informatics, Graduate School of Information Science and \\ Technology, Hokkaido University, Sapporo 060-0814, Japan \\ E-mail: sugawara@ist.hokudai.ac.jp
}

\begin{abstract}
A propagator method (PM), a numerical technique to solve the Boltzmann equation (BE) for the electron velocity or energy distribution function (EVDF/EEDF) of electron swarms in gases, was customized to obtain the equilibrium solution quickly. The PM calculates the number of electrons in cells defined in velocity space using an operator called the propagator or Green's function. The propagator represents the intercellular transfer of electrons corresponding to the electron velocity change due to the acceleration by the electric field and the collisional events with gas molecules. The relaxation of the EVDF to its drift equilibrium solution proceeds with iterative propagator operations for the EVDF. Merits of the PM are that the series expansion of the EVDF as done in the BE analyses is not required and that time evolution of the electron swarm can be observed if necessary. On the other hand, in case only the equilibrium solution of the EVDF is wanted, the relaxation can be accelerated numerically. A demonstration achieved a shortening of the computational time by about three orders of magnitude. Furthermore, this scheme was applied to calculations of a set of electron transport parameters required in fluid-model simulations, i.e. the effective ionization frequency, the centroid drift velocity and the longitudinal diffusion coefficient, using the zeroth-, first- and second-order moment equations derived from the BE. A detailed description on the PM calculation was presented.
\end{abstract}

PACS numbers: 52.65.-y Plasma simulation, 52.20.-j Elementary processes in plasma, 52.25.Fi Transport properties

$\ddagger$ Published source: Plasma Sources Science and Technology 26(4) 044002 (16pp) (2017)

DOI: $10.1088 / 1361-6595 /$ aasd7f 


\section{Introduction}

The electron velocity or energy distribution function (EVDF or EEDF) of electron swarm in gases is one of the most fundamental properties of electric discharges and plasmas. The electron transport parameters derived from the EVDF describe spatial development of plasmas, and the EEDF governs electron interactions with gas molecules in production of reactive species.

The Boltzmann equation (BE) analysis is a traditional solution method of the EVDF/EEDF under dc electric fields. A typical approach is a series expansion of the EVDF using spherical harmonic function. Holstein (1946) expanded the BE using the Legendre polynomials and solved the derived simultaneous differential equations for the zeroth and first order terms of the EVDF, i.e. the isotropic and directional terms. This two-term approximation is prevailing today as an established calculation method of the EVDF. When the anisotropy of the EVDF is significant, we need to consider

higher-order terms. Yachi et al $(1988,1991)$ performed a six-term BE analysis using the Galerkin method. Loffhagen and Winkler (1996) developed a calculation scheme treating the expansion terms being time-dependent in a BE analysis with six to eight terms. Furthermore, Boyle et al (2015) treated the number of expansion terms as a variable to confirm the convergence of solution. On the other hand, spatial electron distribution in an electron swarm can be expressed by a Fourier integral (Parker and Lowke 1969, Tagashira et al 1977, and Kumar et al 1980). Date et al (1992) and Tagashira (1992) applied the Fourier transform to the BE to obtain the dispersion function of the electron swarm development, and definitions of the electron transport parameters dependent on the observation mode were discussed.

The propagator method (PM), presented in this paper, is a numerical technique to solve the BE. The PM simulates kinetic behavior of electrons without the series expansion mentioned above. In the PM, the electron motion is expressed as electron flow from a cell defined in velocity space to other cells by an operator called the propagator or Green's function. The principle of the PM for the calculation of the EVDF in gases has been established by the 1990s. Drallos and Wadehra $(1988,1989)$ demonstrated a timedependent evolution of the EVDF in a gas. Sommerer et al (1989, 1991), Mankelevich et al (1991) Sugawara et al (1992), Hitchon et al (1993), Parker et al (1993), and Wichaidit and Hitchon (2005) simulated spatial development of electrons between parallel plane electrodes or in one-dimensional real space. The PM was also applied to ion transport (Hitchon et al 1989), propagation of excited species and radiation (Tan et al 1996, Christlieb et al 2009, Wichaidit et al 2009 and Golubovskii et al 2005, 2013), and carrier transport in semiconductor devices (Fixel and Hitchon 2007). Sugawara et al (1994) modified the PM to calculate the EEDF in a boundary-free steady-state Townsend mode. The PM was effective in calculation of backward electron diffusion in steady-state electron flow from a point source (Sugawara et al 1995). Furthermore, the PM was extended to obtain the real-space drift velocity $W_{\mathrm{r}}$ (Sugawara et al 1997), the longitudinal diffusion coefficient $D_{\mathrm{L}}$ and its higher-order coefficients $D_{k}(k \geq 3)$ 
(Sugawara et al 1998), and the transverse diffusion coefficients $D_{\mathrm{T}}$ and its higher-order coefficients $D_{\mathrm{T} k}(k \geq 3)$ (Sugawara et al 1999).

Computational methods known with similar or related concepts are the direct estimation of moment (Kitamori et al 1980), the path integral method (Skullerud and Kuhn 1983, Segur et al 1984), and the flight time integral method (Ikuta and Murakami 1987, Ikuta et al 1988). Their characteristics were compared in a survey by Segur et al (1986) and in a discussion on the flight time integral method by Kumar (1995).

The PM requires huge arrays of variables and a heavy computational load. This was a restriction in the early decades. However, nowadays, the computational resources have been enriched drastically both in the capacity and the speed. It would be meaningful to re-evaluate the advantage of the PM such as simple and intuitive treatment on the kinetic motion of electrons and numerical stability under the current computational environment.

This paper presents a detailed configuration of the PM calculation. The EEDF in a gas was calculated as a demonstration. An acceleration scheme to obtain the drift equilibrium solution of the EVDF quickly was newly introduced. Furthermore, this acceleration technique was applied to an extended PM for moment equations derived from the BE for a set of electron transport parameters necessary in one-dimensional fluid-model simulations.

\section{Simulation model and target properties}

We consider electron swarm development along the $x$-axis under a uniform electric field $\boldsymbol{E}=\left(E_{x}, E_{y}, E_{z}\right)=(-E, 0,0)(E>0)$. The EVDF of the electron swarm is the target property, from which fundamental electron swarm parameters, or transport coefficients, such as the mean electron energy and the drift velocity are derived. We assume that the initial electrons are released from the origin with a given initial EVDF. The kinetic electron motion in real space is relevantly considered in the calculation but the electron position is not cared in the final result.

The electron motion is described with the position $\boldsymbol{r}=(x, y, z)$, velocity $\boldsymbol{v}=$ $\left(v_{x}, v_{y}, v_{z}\right)$ and time $t$. $\boldsymbol{v}$ may be represented by polar coordinates $(v, \theta, \phi)$, where $v=|\boldsymbol{v}|$, $\theta$ is the polar angle between the $+x$ direction and $\boldsymbol{v}$, and $\phi$ is the azimuthal angle around the $x$-axis. $v_{x}=v \cos \theta, v_{y}=v_{\perp} \cos \phi, v_{z}=v_{\perp} \sin \phi$ and $v_{\perp}=v \sin \theta=\sqrt{v_{y}^{2}+v_{z}^{2}}$. Axial symmetry around the $x$-axis and uniformity of the EVDF with respect to $\phi$ are assumed.

\section{Electron velocity distribution function and the Boltzmann equation}

The BE describing the electron swarm development is

$$
\frac{\partial}{\partial t} f(\boldsymbol{r}, \boldsymbol{v}, t)=\left[-\boldsymbol{a} \cdot \frac{\partial}{\partial \boldsymbol{v}}-\boldsymbol{v} \cdot \frac{\partial}{\partial \boldsymbol{r}}+\left(\frac{\partial}{\partial t}\right)_{\text {coll }}\right] f(\boldsymbol{r}, \boldsymbol{v}, t),
$$

where $f(\boldsymbol{r}, \boldsymbol{v}, t)=f\left(x, y, z, v_{x}, v_{y}, v_{z}, t\right)$ or $f(x, y, z, v, \theta, \phi, t)$ is the electron distribution function representing the number density of electrons in phase space $(\boldsymbol{r}, \boldsymbol{v})$ at time $t$, 
$\boldsymbol{a}=(a, 0,0)=-e \boldsymbol{E} / m=(e E / m, 0,0)$ is the electron acceleration by $\boldsymbol{E}$, and $e$ and $m$ are the electronic charge and mass, respectively. $(\partial / \partial t)_{\text {coll }}$ is the collision operator representing the changes of the electron population and velocity due to collisional events.

By integrating equation (1) over $\boldsymbol{r}$ and $\phi$, we obtain

$$
\frac{\partial}{\partial t} f(v, \theta, t)=\left[-a \frac{\partial}{\partial v_{x}}+\left(\frac{\partial}{\partial t}\right)_{\text {coll }}\right] f(v, \theta, t) .
$$

Here, the dimension of the EVDF has been reduced on the basis of the disregard of electron position and the axial symmetry in velocity space as

$$
\begin{aligned}
f(v, \theta, \phi, t) & =\int_{x=-\infty}^{\infty} \int_{y=-\infty}^{\infty} \int_{z=-\infty}^{\infty} f(x, y, z, v, \theta, \phi, t) \mathrm{d} x \mathrm{~d} y \mathrm{~d} z \\
f(v, \theta, t) & =\int_{0}^{2 \pi} f(v, \theta, \phi, t) v \sin \theta \mathrm{d} \phi .
\end{aligned}
$$

Note that the space where an EVDF $f$ is defined is distinguished by its arguments; e.g., the physical dimension of $f(v, \theta, \phi, t)$ is (speed) $)^{-3}$ and that of $f(v, \theta, t)$ is (speed $)^{-2}$.

The two operators in the right-hand side of equation (2) are described as the propagators of acceleration and collision, respectively, in the following section.

\section{Calculation scheme of propagator method}

\subsection{Cells}

Two-variable velocity space $(v, \theta)$ is divided into cells for calculation of the number of electrons within each cell. An overview and the specification of the cells defined below are summarized in figure 1 and table 1.

The cells are defined for every $\Delta \varepsilon$ and $\Delta \theta$, where $\varepsilon=\frac{1}{2} m v^{2}$ is the electron energy. The $(i, j)$ th cell $\mathrm{C}_{i, j}\left(0 \leq i<i_{\text {ceiling }}\right.$ and $\left.0 \leq j<j_{\text {ceiling }}\right)$ occupies the region of $v_{i} \leq v<v_{i+1}$ (corresponding to $\varepsilon_{i} \leq \varepsilon<\varepsilon_{i+1}$ ) and $\theta_{j} \leq \theta<\theta_{j+1}$, where

$v_{i}=v_{1 \mathrm{eV}} \sqrt{\varepsilon_{i} / \varepsilon_{1 \mathrm{eV}}}, \varepsilon_{i}=i \Delta \varepsilon, \Delta \varepsilon=\varepsilon_{\max } / i_{\text {ceiling }}, \theta_{j}=j \Delta \theta, \Delta \theta=\pi / j_{\text {ceiling }}, v_{1 \mathrm{eV}}$ is the electron speed associated with $1 \mathrm{eV}, \varepsilon_{1 \mathrm{eV}}=\frac{1}{2} m v_{1 \mathrm{eV}}^{2}=1 \mathrm{eV}$ and $\varepsilon_{\max }$ is the upper limit of the EEDF calculation range. The cell division is nonuniform with respect to $v$. The division with a constant $\Delta \varepsilon$ makes the treatment of the electron energy loss due to inelastic collisions simple as shown afterward. $\Delta \varepsilon, \Delta \theta$ and $\varepsilon_{\max }$ are chosen empirically depending on the required resolution and calculation range for the EEDF. A possible measure for $\Delta \varepsilon$ is whether the precision of the inelastic energy loss discretized by $\Delta \varepsilon$ is satisfactory or not. $\Delta \theta$ is chosen by trial PM calculations. A sufficiently fine value of $\Delta \theta$ would be found by decreasing $\Delta \theta$ until certain directional parameters such as the average velocity in the direction of $\boldsymbol{E}$ becomes unchanged in required significant digits. Their practical values are specified in every demonstration.

Here, as properties of $\mathrm{C}_{i, j}$ necessary to consider the intercellular electron transfer, we define some terms and quantities.

The cells in the regions of $0 \leq \theta<\frac{1}{2} \pi\left(v_{x}>0\right)$ and $\frac{1}{2} \pi \leq \theta<\pi\left(v_{x} \leq 0\right)$ are named the forward and backward cells, respectively. The boundaries of $\mathrm{C}_{i, j}$ contacting with its 


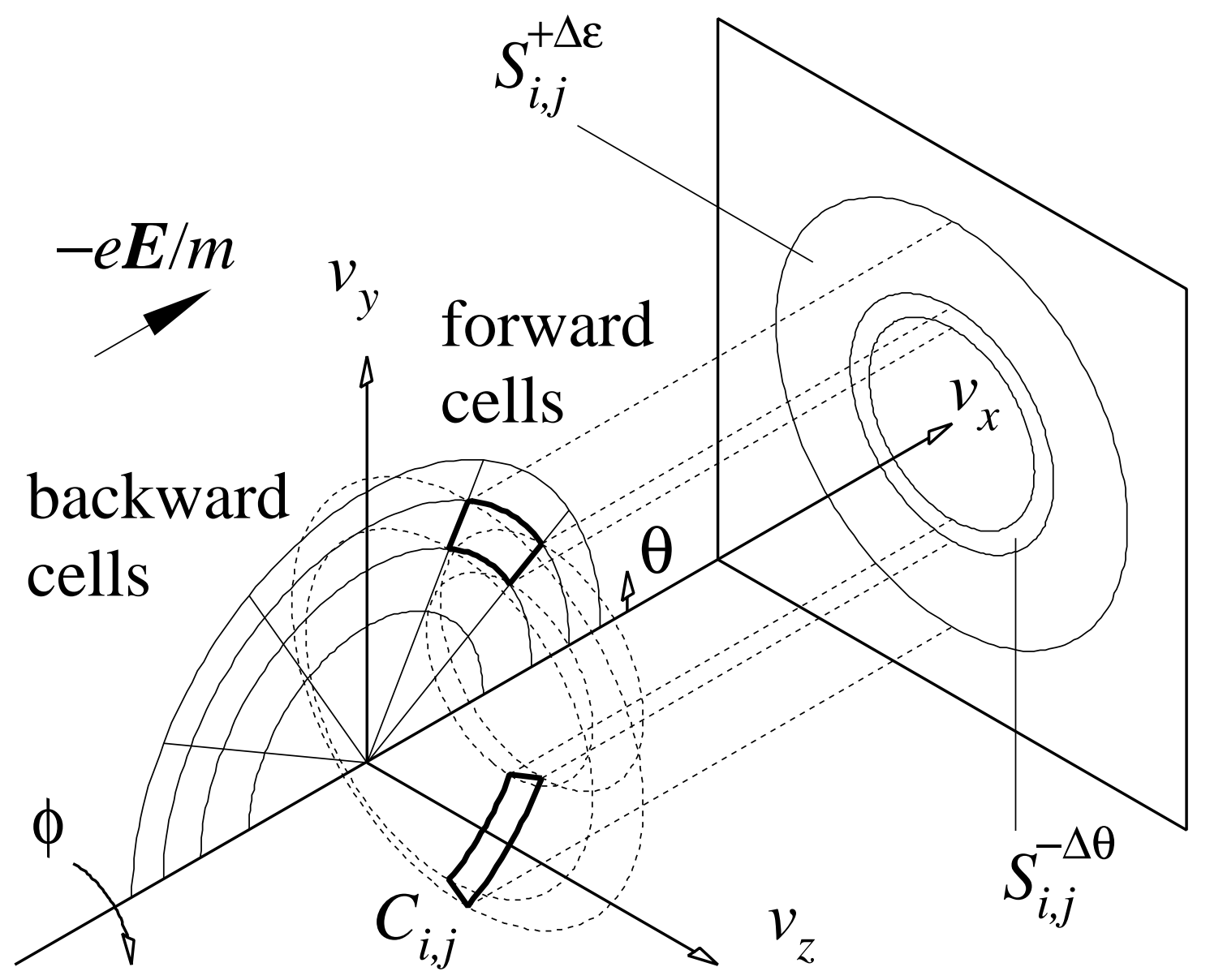

Figure 1. Cells defined in velocity space $\left(v_{x}, v_{y}, v_{z}\right)$. The $(i, j)$ th cell $\mathrm{C}_{i, j}$ occupies the region of $v_{1 \mathrm{eV}} \sqrt{i \Delta \varepsilon / \varepsilon_{1 \mathrm{eV}}} \leq v<v_{1 \mathrm{eV}} \sqrt{(i+1) \Delta \varepsilon / \varepsilon_{1 \mathrm{eV}}}$ and $j \Delta \theta \leq \theta<(j+1) \Delta \theta$.

neighbours $\mathrm{C}_{i, j-1}, \mathrm{C}_{i+1, j}$ and $\mathrm{C}_{i-1, j}$ are denoted as $\mathrm{B}_{i, j}^{-\Delta \theta}, \mathrm{B}_{i, j}^{+\Delta \varepsilon}$ and $\mathrm{B}_{i, j}^{-\Delta \varepsilon}$, respectively. Two of them are facing to the $+v_{x}$ direction. They are $\mathrm{B}_{i, j}^{-\Delta \theta}$ and $\mathrm{B}_{i, j}^{+\Delta \varepsilon}$ when $\mathrm{C}_{i, j}$ is a forward cell of $j>0$, and are $\mathrm{B}_{i, j}^{-\Delta \theta}$ and $\mathrm{B}_{i, j}^{-\Delta \varepsilon}$ when $\mathrm{C}_{i, j}$ is a backward cell of $i>0$. Let us call them the downstream boundaries of $\mathrm{C}_{i, j}$. Hereafter, when they are mentioned, the sign in the superscript of $\mathrm{B}_{i, j}^{ \pm \Delta}$ and concerning properties is to be chosen depending on the region to which $\mathrm{C}_{i, j}$ under consideration belongs. Note that $\mathrm{B}_{i, 0}^{-\Delta \theta}$ and $\mathrm{B}_{0, j}^{-\Delta \varepsilon}$ are not considered because $\mathrm{C}_{i,-1}$ and $\mathrm{C}_{-1, j}$ are not present.

Let us define the cell volume $V_{i, j}$, projected areas $S_{i, j}^{-\Delta \theta}$ of $\mathrm{B}_{i, j}^{-\Delta \theta}$ and $S_{i, j}^{ \pm \Delta \varepsilon}$ of $\mathrm{B}_{i, j}^{ \pm \Delta \varepsilon}$ on the $v_{y} v_{z}$-plane, and the solid angle $\Omega_{j}$ subtended at $\boldsymbol{v}=0$ as properties of $\mathrm{C}_{i, j}$ :

$$
\begin{aligned}
V_{i, j} & =\int_{v=v_{i}}^{v_{i+1}} \int_{\theta=\theta_{j}}^{\theta_{j+1}} \int_{\phi=0}^{2 \pi} v^{2} \sin \theta \mathrm{d} v \mathrm{~d} \theta \mathrm{d} \phi \\
& =\frac{2}{3} \pi\left(v_{i+1}^{3}-v_{i}^{3}\right)\left(\cos \theta_{j}-\cos \theta_{j+1}\right),
\end{aligned}
$$


Table 1. Properties of the $(i, j)$ th cell $\mathrm{C}_{i, j}$ defined in velocity space $\left(v_{x}, v_{y}, v_{z}\right)$.

\begin{tabular}{|c|c|}
\hline property & value or range \\
\hline electron energy & $\varepsilon_{i} \leq \varepsilon<\varepsilon_{i+1}, \varepsilon_{i}=i \Delta \varepsilon(\mathrm{eV})$ \\
\hline representative electron energy & $\varepsilon_{i}^{\mathrm{R}}=\frac{1}{2}\left(\varepsilon_{i}+\varepsilon_{i+1}\right)(\mathrm{eV})$ \\
\hline \multirow[t]{2}{*}{ electron speed } & $v_{i} \leq v<v_{i+1}\left(\mathrm{~m} \mathrm{~s}^{-1}\right)$ \\
\hline & $v_{i}=v_{1 \mathrm{eV}} \sqrt{\varepsilon_{i} / \varepsilon_{1 \mathrm{eV}}}, \varepsilon_{1 \mathrm{eV}}=\frac{1}{2} m v_{1 \mathrm{eV}}^{2}=1 \mathrm{eV}$ \\
\hline representative electron speed & $v_{i}^{\mathrm{R}}=v_{1 \mathrm{eV}} \sqrt{\varepsilon_{i}^{\mathrm{R}} / \varepsilon_{1 \mathrm{eV}}}\left(\mathrm{m} \mathrm{s}^{-1}\right)$ \\
\hline polar angle & $\theta_{j} \leq \theta<\theta_{j+1}, \theta_{j}=j \Delta \theta(\mathrm{rad})$ \\
\hline representative polar angle & $\theta_{j}^{\mathrm{R}}=\frac{1}{2}\left(\theta_{j}+\theta_{j+1}\right)(\mathrm{rad})$ \\
\hline volume & $V_{i, j}=\frac{2}{3} \pi\left(v_{i+1}^{3}-v_{i}^{3}\right)\left(\cos \theta_{j}-\cos \theta_{j+1}\right)\left(\mathrm{m}^{3} \mathrm{~s}^{-3}\right)$ \\
\hline projected area & $\begin{array}{l}S_{i, j}^{+\Delta \varepsilon}=\pi v_{i+1}^{2}\left(\sin ^{2} \theta_{j+1}-\sin ^{2} \theta_{j}\right)\left(\mathrm{m}^{2} \mathrm{~s}^{-2}\right) \\
\left(\mathrm{C}_{i, j}-\mathrm{C}_{i+1, j} \text { boundary for } 0 \leq \theta<\frac{1}{2} \pi\right)\end{array}$ \\
\hline projected area & $\begin{array}{l}S_{i, j}^{-\Delta \varepsilon}=\pi v_{i}^{2}\left(\sin ^{2} \theta_{j}-\sin ^{2} \theta_{j+1}\right)\left(\mathrm{m}^{2} \mathrm{~s}^{-2}\right) \\
\left(\mathrm{C}_{i, j}-\mathrm{C}_{i-1, j} \text { boundary for } \frac{1}{2} \pi \leq \theta<\pi\right)\end{array}$ \\
\hline projected area & $S_{i, j}^{-\Delta \theta}=\pi\left(v_{i+1}^{2}-v_{i}^{2}\right) \sin ^{2} \theta_{j}\left(\mathrm{~m}^{2} \mathrm{~s}^{-2}\right)$ \\
\hline & $\left(\mathrm{C}_{i, j}-\mathrm{C}_{i, j-1}\right.$ boundary for $\left.0 \leq \theta<\pi\right)$ \\
\hline solid angle subtended at $\boldsymbol{v}=0$ & $\Omega_{j}=2 \pi\left(\cos \theta_{j}-\cos \theta_{j+1}\right)(\mathrm{sr})$ \\
\hline number of electrons & $n_{i, j}=\int_{v=v_{i}}^{v_{i+1}} \int_{\theta=\theta_{j}}^{\theta_{j+1}} \int_{\phi=0}^{2 \pi} \int_{\boldsymbol{r}} f(\boldsymbol{r}, \boldsymbol{v}) \mathrm{d} \boldsymbol{r} \mathrm{d} \boldsymbol{v}$ \\
\hline electron number density & $n_{i, j} / V_{i, j}\left(\mathrm{~m}^{-3} \mathrm{~s}^{3}\right)$ \\
\hline outflowing electron flux & $\Gamma_{i, j}=a n_{i, j} / V_{i, j}\left(\mathrm{~m}^{-2} \mathrm{~s}\right)$ \\
\hline zeroth-order moment & $m_{0, i, j}=n_{i, j}$ \\
\hline first-order moment & $m_{1, i, j}=\int_{v=v_{i}}^{v_{i+1}} \int_{\theta=\theta_{j}}^{\theta_{j+1}} \int_{\phi=0}^{2 \pi} \int_{\boldsymbol{r}} x f(\boldsymbol{r}, \boldsymbol{v}) \mathrm{d} \boldsymbol{r} \mathrm{d} \boldsymbol{v}(\mathrm{m})$ \\
\hline second-order moment & $m_{2, i, j}=\int_{v=v_{i}}^{v_{i+1}} \int_{\theta=\theta_{j}}^{\theta_{j+1}} \int_{\phi=0}^{2 \pi} \int_{\boldsymbol{r}} x^{2} f(\boldsymbol{r}, \boldsymbol{v}) \mathrm{d} \boldsymbol{r} \mathrm{d} \boldsymbol{v}\left(\mathrm{m}^{2}\right)$ \\
\hline
\end{tabular}

$$
\begin{aligned}
& S_{i, j}^{-\Delta \theta}=\pi\left(v_{i+1}^{2}-v_{i}^{2}\right) \sin ^{2} \theta_{j}, \\
& S_{i, j}^{+\Delta \varepsilon}=\pi v_{i+1}^{2}\left(\sin ^{2} \theta_{j+1}-\sin ^{2} \theta_{j}\right), \\
& S_{i, j}^{-\Delta \varepsilon}=\pi v_{i}^{2}\left(\sin ^{2} \theta_{j}-\sin ^{2} \theta_{j+1}\right), \\
& \Omega_{j}=\int_{\theta=\theta_{j}}^{\theta_{j+1}} \int_{\phi=0}^{2 \pi} \sin \theta \mathrm{d} \theta \mathrm{d} \phi=2 \pi\left(\cos \theta_{j}-\cos \theta_{j+1}\right) .
\end{aligned}
$$

The dimensions of $V_{i, j}$ and $S_{i, j}$ are (speed) ${ }^{3}$ and (speed) $)^{2}$, respectively.

$\mathrm{C}_{i, j}$ has a value of the number of electrons in it, $n_{i, j}(t)$ :

$$
n_{i, j}(t)=\int_{v=v_{i}}^{v_{i+1}} \int_{\theta=\theta_{j}}^{\theta_{j+1}} f(v, \theta, t) v \mathrm{~d} v \mathrm{~d} \theta
$$

Here, the electrons in a cell are assumed to be uniform with respect to the volume element in velocity space. This treatment corresponds to the zeroth-order finite volume method. Change of $n_{i, j}(t)$ is calculated by the acceleration and collision propagators on the basis of equation (2). 
In addition, the representative energy $\varepsilon_{i}^{\mathrm{R}}$, velocity $v_{i}^{\mathrm{R}}$ and polar angle $\theta_{j}^{\mathrm{R}}$ of $\mathrm{C}_{i, j}$ are defined as follows to calculate swarm parameters from the discretized $\operatorname{EVDF} n_{i, j}(t)$ :

$$
\begin{aligned}
\varepsilon_{i}^{\mathrm{R}} & =\frac{1}{2}\left(\varepsilon_{i}+\varepsilon_{i+1}\right), \\
v_{i}^{\mathrm{R}} & =v_{1 \mathrm{eV}} \sqrt{\frac{\varepsilon_{i}^{\mathrm{R}}}{\varepsilon_{1 \mathrm{eV}}}}, \\
\theta_{j}^{\mathrm{R}} & =\frac{1}{2}\left(\theta_{j}+\theta_{j+1}\right) .
\end{aligned}
$$

\subsection{Electron swarm parameters available from the electron velocity distribution} function

We can derive the mean electron energy $\bar{\varepsilon}(t)$, the collision frequency $\nu_{k}(t)$ of the $k$ th kind of reaction and the mean electron velocity $W_{\mathrm{v}}(t)$ from $n_{i, j}(t)$ as

$$
\begin{aligned}
& \bar{\varepsilon}(t)=\frac{\int_{\boldsymbol{v}} \frac{1}{2} m v^{2} f(\boldsymbol{v}, t) \mathrm{d} \boldsymbol{v}}{\int_{\boldsymbol{v}} f(\boldsymbol{v}, t) \mathrm{d} \boldsymbol{v}}=\frac{\sum_{i, j} \varepsilon_{i}^{\mathrm{R}} n_{i, j}(t)}{\sum_{i, j} n_{i, j}(t)}, \\
& \nu_{k}(t)=\frac{\int_{\boldsymbol{v}} N q_{k}(v) v f(\boldsymbol{v}, t) \mathrm{d} \boldsymbol{v}}{\int_{\boldsymbol{v}} f(\boldsymbol{v}, t) \mathrm{d} \boldsymbol{v}}=\frac{\sum_{i, j} N q_{k}\left(v_{i}^{\mathrm{R}}\right) v_{i}^{\mathrm{R}} n_{i, j}(t)}{\sum_{i, j} n_{i, j}(t)}, \\
& W_{\mathrm{v}}(t)=\frac{\int_{\boldsymbol{v}} v_{x} f(\boldsymbol{v}, t) \mathrm{d} \boldsymbol{v}}{\int_{\boldsymbol{v}} f(\boldsymbol{v}, t) \mathrm{d} \boldsymbol{v}}=\frac{\sum_{i, j} v_{i}^{\mathrm{R}} \cos \theta_{j}^{\mathrm{R}} n_{i, j}(t)}{\sum_{i, j} n_{i, j}(t)},
\end{aligned}
$$

where $q_{k}$ is the electron collision cross section of the $k$ th kind of reaction as a function of $v$, and $N$ is the gas molecule number density.

The normalized EEDF $F(\varepsilon, t)\left(\int_{\varepsilon=0}^{\infty} F(\varepsilon, t) \mathrm{d} \varepsilon=1\right)$ and its value at $\varepsilon_{i}^{\mathrm{R}}$ are given as

$$
\begin{aligned}
& F(\varepsilon, t)=\frac{1}{m v} \frac{\int_{\theta=0}^{\pi} \int_{\phi=0}^{2 \pi} f(v, \theta, \phi, t) v^{2} \sin \theta \mathrm{d} \theta \mathrm{d} \phi}{\int_{\boldsymbol{v}} f(\boldsymbol{v}, t) \mathrm{d} \boldsymbol{v}}, \\
& F\left(\varepsilon_{i}^{\mathrm{R}}, t\right)=\frac{1}{\Delta \varepsilon} \frac{\sum_{j} n_{i, j}(t)}{\sum_{i, j} n_{i, j}(t)},
\end{aligned}
$$

where $\sum_{i=0}^{i_{\text {ceiling }}-1} F\left(\varepsilon_{i}^{\mathrm{R}}, t\right) \Delta \varepsilon=1$.

\subsection{Propagator for electron acceleration}

The number of electrons flowing out of $\mathrm{C}_{i, j}$ by the acceleration during a time step $\Delta t$ is calculated here.

The acceleration operator $-a\left(\partial / \partial v_{x}\right)$ in equation $(2)$ represents a parallel shift of electrons in velocity space toward the $+v_{x}$ direction, and its shift speed in velocity space is $a$ (speed/time). The electron outflows through the downstream boundaries $\mathrm{B}_{i, j}^{-\Delta \theta}$ and $\mathrm{B}_{i, j}^{ \pm \Delta \varepsilon}$ become electron inflows for the two downstream neighbor cells $\mathrm{C}_{i, j-1}$ and $\mathrm{C}_{i \pm 1, j}$, respectively. Figure 2 shows typical examples of relationship between source and destination cells in the acceleration process.

The number of electrons, $n_{\text {acc }, i, j}^{\text {out }}$, transferred through the downstream boundaries $\mathrm{B}_{i, j}^{-\Delta \theta}$ and $\mathrm{B}_{i, j}^{ \pm \Delta \varepsilon}$ is obtained as the product of their projected areas, the electron 


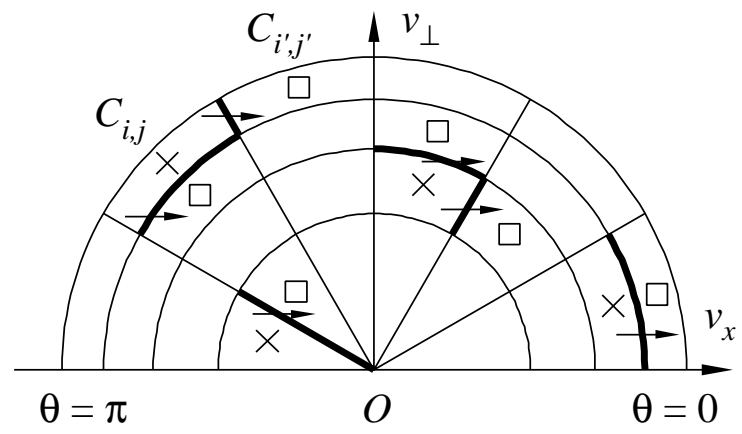

Figure 2. Schematic of electron transfer from source cells $\left(\times: \mathrm{C}_{i, j}\right)$ to destination cells $\left(\square: \mathrm{C}_{i^{\prime}, j^{\prime}}\right)$ due to acceleration. Four typical positions of source cell are shown. The thick boundaries represent the downstream boundaries (i.e. exits) of the source cells.

flux $\Gamma_{i, j}=\left[n_{i, j}(t) / V_{i, j}\right] a\left((\text { speed })^{-2}(\text { time })^{-1}\right)$ in velocity space and the time step $\Delta t$ (Sugawara 1996):

$$
\begin{aligned}
& n_{\mathrm{acc}, i, j}^{\mathrm{out}}=n_{\mathrm{acc}, i, j-1}^{\mathrm{in}}+n_{\mathrm{acc}, i \pm 1, j}^{\mathrm{in}}, \\
& n_{\mathrm{acc}, i, j-1}^{\mathrm{in}}=S_{i, j}^{-\Delta \theta} \Gamma_{i, j} \Delta t=\frac{S_{i, j}^{-\Delta \theta} a \Delta t}{V_{i, j}} n_{i, j}(t), \\
& n_{\mathrm{acc}, i \pm 1, j}^{\mathrm{in}}=S_{i, j}^{ \pm \Delta \varepsilon} \Gamma_{i, j} \Delta t=\frac{S_{i, j}^{ \pm \Delta \varepsilon} a \Delta t}{V_{i, j}} n_{i, j}(t) .
\end{aligned}
$$

$n_{\mathrm{acc}, i, j}^{\text {out }}$ is taken from $\mathrm{C}_{i, j}$, and given to $\mathrm{C}_{i, j-1}$ and $\mathrm{C}_{i \pm 1, j}$ as $n_{\mathrm{acc}, i, j-1}^{\mathrm{in}}$ and $n_{\mathrm{acc}, i \pm 1, j}^{\mathrm{in}}$, respectively.

The electrons flowing out of the most outer forward cells $\mathrm{C}_{i_{\text {ceiling }}-1, j}$ over the upper limit $\varepsilon_{\max }$ of the calculation range are ignored. In order to validate this treatment, $\varepsilon_{\max }$ should be sufficiently high so that $F\left(\varepsilon_{i_{\text {ceiling }}^{\mathrm{R}}-1}^{\mathrm{R}}\right) \ll \max (F(\varepsilon))$.

\subsection{Propagator for collisional events}

The number of electrons to be scattered out of $\mathrm{C}_{i, j}$ by collisions of the $k$ th kind of reaction during $\Delta t$ is $n_{i, j}(t) \nu_{k, i} \Delta t$, where $\nu_{k, i}=N q_{k}\left(v_{i}^{\mathrm{R}}\right) v_{i}^{\mathrm{R}}$. The collision operator $(\partial / \partial t)_{\text {coll }}$ in equation (2) represents redistribution of $\sum_{k} n_{i, j}(t) \nu_{k, i} \Delta t$ from the source cell $\mathrm{C}_{i, j}$ to the relevant destination cells $\mathrm{C}_{i^{\prime}, j^{\prime}}$.

Isotropic scattering in laboratory system is assumed here for simplicity. The intercellular electron transfer due to collisions is treated as the following descriptions. Their aspects are illustrated in figure 3 and the relationship between $\mathrm{C}_{i, j}$ and $\mathrm{C}_{i^{\prime}, j^{\prime}}$ is summarized in table 2 .

4.4.1. Elastic collision Scattering by elastic collisions is treated as change of electron flight direction. The cold gas model is assumed here and the change of electron energy is neglected since the mass ratio $m / M$ between an electron and a gas molecule, that determines the energy loss, is small. 

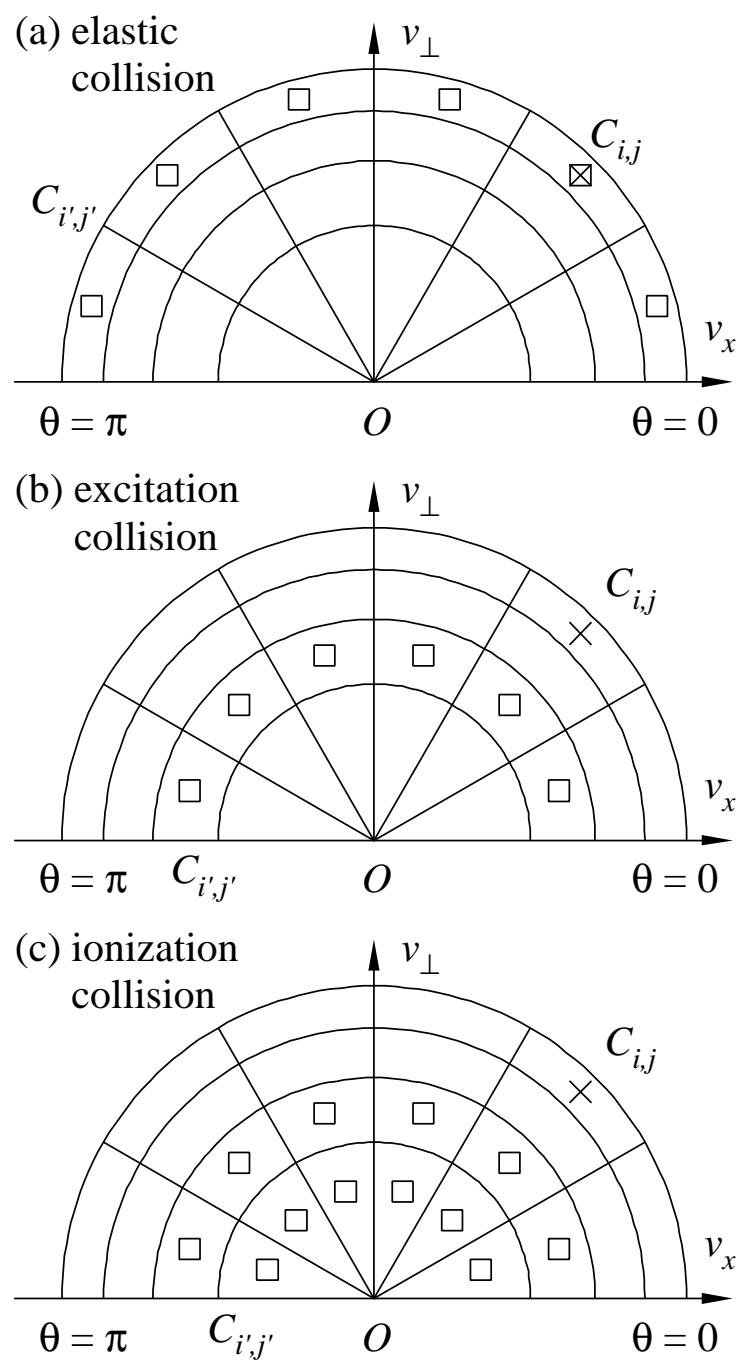

Figure 3. Schematic of relationship between source $\left(\times: C_{i, j}\right)$ and destination $(\square$ : $\left.\mathrm{C}_{i^{\prime}, j^{\prime}}\right)$ cells for electron transfer at collisional events: $(a)$ elastic collision, $i^{\prime}=i ;(b)$ excitation collision, $i^{\prime}=i-k_{\mathrm{ex}}$; and $(c)$ ionization collision, $i^{\prime} \leq i-k_{\text {ion }}$.

$\mathrm{C}_{i, j}$ and $\mathrm{C}_{i^{\prime}, j^{\prime}}$ are in a relationship of $i^{\prime}=i$ and $0 \leq j^{\prime}<j_{\text {ceiling. The number }}$ of scattered electrons, $n_{i, j}(t) \nu_{\text {elastic }, i} \Delta t$, is redistributed to $\mathrm{C}_{i^{\prime}, j^{\prime}}$ with a ratio $\Omega_{j^{\prime}} /(4 \pi)$, in proportion to the solid angle subtended at $\boldsymbol{v}=0 . \quad \mathrm{C}_{i^{\prime}, j^{\prime}}$ receives electrons of $n_{i, j}(t) \nu_{\text {elastic }, i} \Delta t \times \Omega_{j^{\prime}} /(4 \pi)$ from $\mathrm{C}_{i, j}$ as a result of the isotropic scattering.

In case it is required to consider the energy loss in a light gas, the electron redistribution may be modified so that the cells of $\mathrm{C}_{i^{\prime}-1, j^{\prime}}$ receive a part of the scattered electrons to represent a little decrease of the electron energy. Furthermore, when the thermal motion of gas molecules induces an electron energy increase after scattering, the cells of $\mathrm{C}_{i^{\prime}+1, j^{\prime}}$ receive a part of the scattered electrons at the redistribution. Evaluation of the electron redistribution under these effects is complicated because $\nu_{\text {elastic }, i}$ would be derived from the relative velocity distribution between electrons and gas molecules, 
Table 2. The relationship between source cell $\mathrm{C}_{i, j}$ and destination cell $\mathrm{C}_{i^{\prime}, j^{\prime}}$ for electron transfer at collisional events.

\begin{tabular}{lll}
\hline reaction & relationship & assumption or remark \\
\hline elastic collision & $i^{\prime}=i$ & little energy loss \\
excitation collision & $i^{\prime}=i-k_{\mathrm{ex}}$ & $k_{\mathrm{ex}}=\left\lfloor\varepsilon_{\mathrm{ex}} / \Delta \varepsilon+\frac{1}{2}\right\rfloor$ \\
same treatment for dissociation \\
ionization collision & $i^{\prime} \leq i-k_{\text {ion }}$ & $\begin{array}{l}k_{\text {ion }}=\left\lfloor\varepsilon_{\text {ion }} / \Delta \varepsilon+\frac{1}{2}\right\rfloor \\
\text { number of scattered electrons doubled }\end{array}$ \\
electron attachment & no $\mathrm{C}_{i^{\prime}, j^{\prime}}$ & lost from velocity space \\
\hline scattering & $0 \leq j^{\prime}<j_{\text {ceiling }}$ & isotropic, redistribution ratio $\propto \Omega_{j^{\prime}}$ \\
\hline
\end{tabular}

isotropic scattering would be assumed in the centroid system, and the electron energy loss/gain depends not only on $\theta$ and $\theta^{\prime}$ but also on $\phi$ and $\phi^{\prime}$ before and after the scattering, respectively. However, these factors could be taken into account as average values under some approximation in practice.

4.4.2. Excitation collision At an excitation collision, the electron energies $\varepsilon$ and $\varepsilon^{\prime}$ before and after the loss of the excitation energy $\varepsilon_{\text {ex }}$, respectively, are related as $\varepsilon^{\prime}=\varepsilon-\varepsilon_{\mathrm{ex}}$. This category includes vibrational and rotational excitations. Neutral dissociation and ion pair production in multiatomic molecular gases are also treated in the same way because electron energy loss occurs and the electron population is unchanged in these processes.

$\mathrm{C}_{i, j}$ and $\mathrm{C}_{i^{\prime}, j^{\prime}}$ are related as $i^{\prime}=i-k_{\mathrm{ex}}$, where $k_{\mathrm{ex}}=\left\lfloor\varepsilon_{\mathrm{ex}} / \Delta \varepsilon+\frac{1}{2}\right\rfloor$ represents the energy loss discretized by $\Delta \varepsilon$ (see figure 4(a)). $\lfloor x\rfloor$ represents rounding fractions off and $\left\lfloor x+\frac{1}{2}\right\rfloor$ is the nearest integer to $x$. The redistribution of electrons to $\mathrm{C}_{i^{\prime}, j^{\prime}}$ of different $j^{\prime} \mathrm{s}$ is in the same way as for the elastic collision.

As a finer treatment for the energy loss, the electron redistribution may be made for the cells of $\mathrm{C}_{i^{\prime}, j^{\prime}}$ and $\mathrm{C}_{i^{\prime}-1, j^{\prime}}$ modifying the calculation of $k_{\mathrm{ex}}$ as $k_{\mathrm{ex}}=\left\lfloor\varepsilon_{\mathrm{ex}} / \Delta \varepsilon\right\rfloor$. With the redistribution ratio $\delta_{\mathrm{ex}}^{i \rightarrow i^{\prime}}: \delta_{\mathrm{ex}}^{i \rightarrow i^{\prime}-1}$ for the cells of $\mathrm{C}_{i^{\prime}, j^{\prime}}$ and $\mathrm{C}_{i^{\prime}-1, j^{\prime}}$ given as follows, the average of the energy loss calculated with $\varepsilon_{i}^{\mathrm{R}}$, $\varepsilon_{i^{\prime}}^{\mathrm{R}}$ and $\varepsilon_{i^{\prime}-1}^{\mathrm{R}}$ agrees with $\varepsilon_{\mathrm{ex}}$ (figure 4(b)):

$$
\delta_{\mathrm{ex}}^{i \rightarrow i^{\prime}}: \delta_{\mathrm{ex}}^{i \rightarrow i^{\prime}-1}=1-\left(\frac{\varepsilon_{\mathrm{ex}}}{\Delta \varepsilon}-\left\lfloor\frac{\varepsilon_{\mathrm{ex}}}{\Delta \varepsilon}\right\rfloor\right): \frac{\varepsilon_{\mathrm{ex}}}{\Delta \varepsilon}-\left\lfloor\frac{\varepsilon_{\mathrm{ex}}}{\Delta \varepsilon}\right\rfloor .
$$

4.4.3. Ionization collision Assume that primary electrons with energy $\varepsilon$ undergoes ionization in $\mathrm{C}_{i, j}$ and produce secondary electrons. This process includes dissociative ionization of multiatomic molecular gases. The total number of the primary and secondary electrons is $2 n_{i, j}(t) \nu_{\text {ion }, i} \Delta t$.

Let the energies of a pair of primary and secondary electrons after ionization collision be $\varepsilon^{\prime}$ and $\varepsilon^{\prime \prime}$. They satisfy $\varepsilon^{\prime}+\varepsilon^{\prime \prime}=\varepsilon-\varepsilon_{\text {ion }}$, where $\varepsilon_{\text {ion }}$ is the ionization 

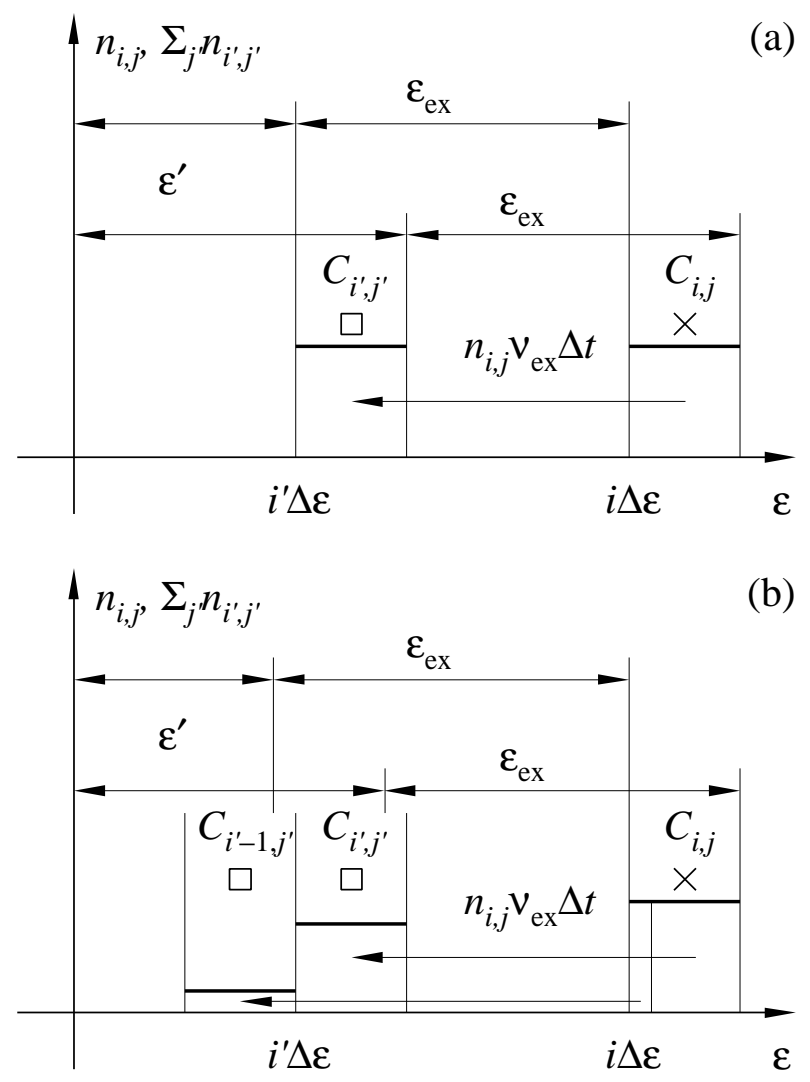

Figure 4. Schematic of electron redistribution from source cell $\left(\times: \mathrm{C}_{i, j}\right)$ to destination cells $\left(\square: \mathrm{C}_{i^{\prime}, j^{\prime}}\right.$ and $\left.\mathrm{C}_{i^{\prime}-1, j^{\prime}}\right)$ by excitation. (a) simplified treatment for the case energy loss can be discretized by $\Delta \varepsilon$ and (b) finer treatment for energy conservation.

potential. The residual energy $\varepsilon-\varepsilon_{\text {ion }}$ is shared by the primary and secondary electrons. The division ratio $\varepsilon^{\prime}: \varepsilon^{\prime \prime}$ is assumed to be uniform between $0: 1$ and $1: 0$ for simplicity. $i^{\prime}$ of $\mathrm{C}_{i^{\prime}, j^{\prime}}$ satisfies $i^{\prime} \leq i-k_{\text {ion }}$, where $k_{\text {ion }}=\left\lfloor\varepsilon_{\text {ion }} / \Delta \varepsilon+\frac{1}{2}\right\rfloor$. Let $\delta_{\text {ion }}^{i \rightarrow i^{\prime}}$ be the ratio of the redistribution of the $2 n_{i, j}(t) \nu_{\mathrm{ion}, i} \Delta t$ electrons for the cells $\mathrm{C}_{i^{\prime}, j^{\prime}}$ of an $i^{\prime}$ and all $j^{\prime} . \delta_{\mathrm{ion}}^{i \rightarrow i^{\prime}}$ is approximated as

$$
\begin{array}{ll}
\delta_{\text {ion }}^{i \rightarrow i^{\prime}}=\frac{2}{2\left(i-k_{\text {ion }}\right)+1} & \text { for } 0 \leq i^{\prime}<i-k_{\text {ion }}, \\
\delta_{\text {ion }}^{i \rightarrow i^{\prime}}=\frac{1}{2\left(i-k_{\text {ion }}\right)+1} & \text { for } i^{\prime}=i-k_{\text {ion }} .
\end{array}
$$

Here, $\sum_{i^{\prime}=0}^{i-k_{\text {ion }}} \delta_{\text {ion }}^{i \rightarrow i^{\prime}}=1$. This approximation is based on a consideration on the range of $i^{\prime}$, which is $0 \leq i^{\prime} \leq i-k_{\text {ion }}$ for an ionization at $\varepsilon=i \Delta \varepsilon$ (i.e. at the lowest energy within $\left.\mathrm{C}_{i, j}\right)$ and $0 \leq i^{\prime} \leq i-k_{\text {ion }}+1$ for that at $(i+1) \Delta \varepsilon$ (the highest energy within $\mathrm{C}_{i, j}$ ) as illustrated in figure 5 (Sugawara 1996). $\mathrm{C}_{i^{\prime}, j^{\prime}}$ receives $2 n_{i, j}(t) \nu_{\mathrm{ion}, i} \Delta t \times \delta_{\text {ion }}^{i \rightarrow i^{\prime}} \Omega_{j^{\prime}} /(4 \pi)$ electrons from $\mathrm{C}_{i, j}$ as a result of the isotropic scattering after ionization.

4.4.4. Electron attachment Electrons undergoing electron attachment or dissociative attachment in $\mathrm{C}_{i, j}$ disappear from velocity space, because they are excluded from the 


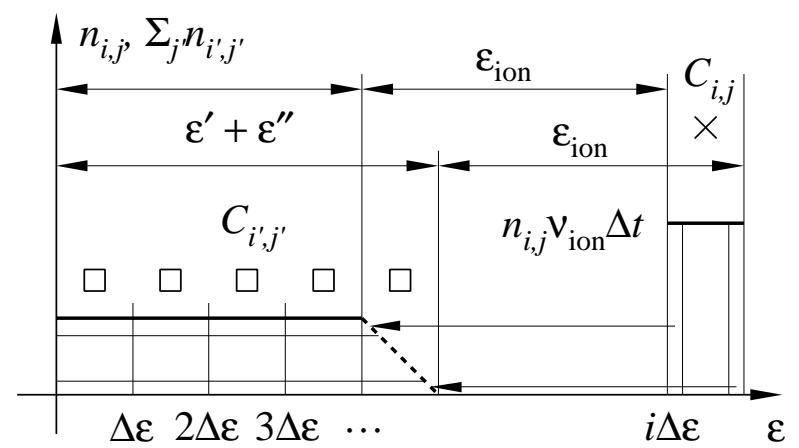

Figure 5. Schematic of electron redistribution from source cell $\left(\times: \mathrm{C}_{i, j}\right)$ to destination cells $\left(\square: \mathrm{C}_{i^{\prime}, j^{\prime}}\right)$ by ionization.

electron swarm. $n_{i, j}(t) \nu_{\text {att }, i} \Delta t$ is simply subtracted from $n_{i, j}(t)$.

\subsection{Physical relaxation of the electron velocity distribution function}

One of the solution techniques of the PM is iterative operation of the acceleration and collision propagators for the EVDF. The EVDF relaxes every $\Delta t$ and the normalized EVDF eventually reaches the drift equilibrium solution. This process is the physical relaxation following the temporal development of the electron swarm. This scheme is applicable not only to the relaxation under dc $\boldsymbol{E}$ fields but also to that under radio-frequency electric fields (e.g., Maeda and Makabe 1994, Shimada et al 2003) and impulse electric fields (Sugawara et al 2003) by changing $a$ in the acceleration propagator temporally.

The relaxation time required to reach the drift equilibrium depends on the physical condition such as $N$ and $E$. Empirically, it becomes shorter at higher $N$ and $E$. An appropriate observation time $T_{\text {obs }}$ is chosen to be sufficiently long for the electron swarm to reach its equilibrium. Preliminary trials are needed for the estimation of $T_{\mathrm{obs}}$.

$\Delta t$ is chosen under a restriction that the electron outflow from $\mathrm{C}_{i, j}$ does not exceed $n_{i, j}(t)$ in order to avoid negative values of $n_{i, j}(t)$ for all cells:

$$
n_{i, j}(t) \frac{S_{i, j}^{-\Delta \theta}+S_{i, j}^{ \pm \Delta \varepsilon}}{V_{i, j}} a \Delta t+\sum_{k} n_{i, j}(t) \nu_{k, i} \Delta t \leq n_{i, j}(t) .
$$

The first term is dominant at low pressures, and the restriction becomes

$$
\Delta t \leq \min _{i, j} \frac{V_{i, j}}{a\left(S_{i, j}^{-\Delta \theta}+S_{i, j}^{ \pm \Delta \varepsilon}\right)} .
$$

On the other hand, at a high pressure, $\Delta t$ is restricted by the collision frequency; it is required that the probability $P_{\text {coll }, i}(X \geq 2, \Delta t)$ of two or more collisions for an electron during $\Delta t$ should be negligible:

$$
P_{\text {coll }, i}(X \geq 2, \Delta t) \simeq \frac{1}{2}\left(\sum_{k} \nu_{k, i} \Delta t\right)^{2} \ll 1,
$$




$$
\Delta t \ll \min _{i}\left(\sum_{k} \nu_{k, i}\right)^{-1} .
$$

The computational load is proportional to $i_{\text {ceiling }} j_{\text {ceiling }} T_{\text {obs }} / \Delta t$. Therefore, a $\Delta t$ longer as much as possible is desirable. Practical values of $i_{\text {ceiling }}, j_{\text {ceiling }}, T_{\text {obs }}$ and $\Delta t$ are shown in section 5 .

\section{Demonstration of the propagator method for the electron energy distribution function}

\subsection{Simulation condition}

For a benchmark of the PM, the EVDF in $\mathrm{SF}_{6}$ was calculated at $E / N=200-1000 \mathrm{Td}$ $\left(1 \mathrm{Td}=10^{-21} \mathrm{Vm}^{2}\right)$ at $133 \mathrm{~Pa}(1 \mathrm{Torr})$ at $237 \mathrm{~K}\left(N=3.54 \times 10^{22} \mathrm{~m}^{-3}\right)$. The electron collision cross section set for $\mathrm{SF}_{6}$ was taken from Itoh et al $(1988,1993)$. This set is desirable in the points that the values of the cross sections are given in forms of formulae so that there is no uncertainty in the interpolation and that the set includes all types of collisional processes considered in the PM, i.e., elastic, excitation and ionization collisions and electron attachment.

The configuration was set as follows. $\Delta \varepsilon=0.1 \mathrm{eV}, \Delta \theta=\pi / 180$ and $\varepsilon_{\max }=100 \mathrm{eV}$, i.e. $i_{\text {ceiling }}=1000$ and $j_{\text {ceiling }}=180$. At $E / N=1000 \mathrm{Td}, \Delta t$ must be shorter than about 0.35 ps to satisfy equation (26). On the other hand, $\min _{i}\left(\sum_{k} \nu_{k, i}\right)^{-1}$ in equation (28) was 26.0 ps at the present $N$ of $\mathrm{SF}_{6}$. Let us treat $P_{\text {coll }, i}(X \geq 2, \Delta t)$ being negligible when $P_{\text {coll }, i}(X \geq 2, \Delta t) \leq 10^{-4}$, for example, then the restriction in equation (27) becomes $\Delta t \leq 0.37 \mathrm{ps}$. In the benchmark, $\Delta t$ was set at $0.05 \mathrm{ps}$ leaving a good safety margin.

$T_{\text {obs }}$ was chosen to be $5 \mathrm{~ns}$ at $E / N=1000 \mathrm{Td}$ and $20 \mathrm{~ns}$ at $200 \mathrm{Td}$, until which $\bar{\varepsilon}$ and $\bar{\nu}_{\text {ion }}=\nu_{\text {ion }}-\nu_{\text {att }}$ became invariant in five to six digits of significant figures, where $\bar{\nu}_{\text {ion }}$ is the effective ionization frequency. $\bar{\nu}_{\text {ion }}<0$ (attachment-dominated) at $200 \mathrm{Td}$. The iteration of the propagator operations was $10^{5}$ times for $1.8 \times 10^{5}$ cells for a relaxation of $5 \mathrm{~ns}$.

A workstation with the following specifications was used for the benchmark. CPU, Intel Xeon E5-2667; clock frequency, 2.9 GHz; main memory, $32 \mathrm{~GB}$; operating system, Linux CentOS 6.4; programming language, $\mathrm{C}++$.

Starting from an initial EVDF, the change of $n_{i, j}(t)$ was calculated by operating the acceleration and collision propagators until $t=T_{\text {obs }}$. A Maxwellian EEDF $F_{\mathrm{M}}(\varepsilon)$ was assumed as the initial condition. $n_{i, j}(0)$ was given as

$$
\begin{aligned}
F_{\mathrm{M}}(\varepsilon) & =\frac{2}{\sqrt{\pi}}\left(\frac{1}{k_{\mathrm{B}} T}\right)^{3 / 2} \sqrt{\varepsilon} \exp \left(-\frac{\varepsilon}{k_{\mathrm{B}} T}\right), \\
n_{i, j}(0) & =\frac{\Omega_{j}}{4 \pi} F_{\mathrm{M}}\left(\varepsilon_{i}^{\mathrm{R}}\right) \Delta \varepsilon \\
& =\frac{\Omega_{j}}{4 \pi} \frac{2}{\sqrt{\pi}}\left(\frac{3}{2 \bar{\varepsilon}}\right)^{3 / 2} \sqrt{\varepsilon_{i}^{\mathrm{R}}} \exp \left(-\frac{3 \varepsilon_{i}^{\mathrm{R}}}{2 \bar{\varepsilon}}\right) \Delta \varepsilon,
\end{aligned}
$$




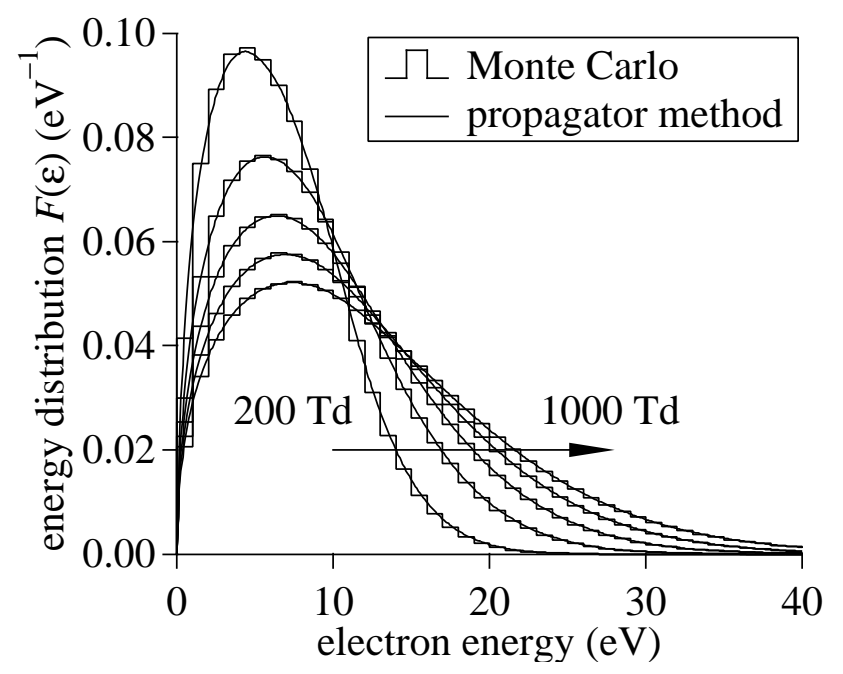

Figure 6. Electron energy distribution $F(\varepsilon)$ in $\mathrm{SF}_{6}$ at $E / N=200,400,600,800$ and $1000 \mathrm{Td}(E=70.7,141.4,212.2,282.9$ and $353.6 \mathrm{~V} / \mathrm{cm}, 133 \mathrm{~Pa}$ at $273 \mathrm{~K})$ : histograms, MC; and curves, PM. The discrepancy $d$ between $F_{\mathrm{PM}}(\varepsilon)$ and $F_{\mathrm{MC}}(\varepsilon)$ defined in equation (32) was $0.00677,0.00413,0.00282,0.00237$ and 0.00205 , respectively.

where $k_{\mathrm{B}}$ is the Boltzmann constant, $T$ is the electron temperature, and $\bar{\varepsilon}=\frac{3}{2} k_{\mathrm{B}} T$ was set at $1 \mathrm{eV}$.

\subsection{Results of the electron energy distribution function}

Figure 6 shows $F\left(\varepsilon, T_{\text {obs }}\right)$ in $\mathrm{SF}_{6}$ calculated by the $\mathrm{PM}$ and a Monte Carlo (MC) simulation, $F_{\mathrm{PM}}(\varepsilon)$ and $F_{\mathrm{MC}}(\varepsilon)$. A sufficient number of electrons, more than $10^{7}$ at $t=T_{\mathrm{obs}}$, were sampled in the MC to reduce the statistical fluctuation.

The discrepancy $d$ between $F_{\mathrm{PM}}(\varepsilon)$ and $F_{\mathrm{MC}}(\varepsilon)$ was evaluated by a norm defined as

$$
d=\frac{1}{2} \int_{0}^{\infty}\left|F_{\mathrm{PM}}(\varepsilon)-F_{\mathrm{MC}}(\varepsilon)\right| \mathrm{d} \varepsilon
$$

Since $F_{\mathrm{PM}}(\varepsilon)$ and $F_{\mathrm{MC}}(\varepsilon)$ have been normalized, $d$ is dimensionless, $d=0$ when $F_{\mathrm{PM}}(\varepsilon)=F_{\mathrm{MC}}(\varepsilon)$, and $d=1$ when they have no overlap. To fit the discretized sampling of the MC, equation (31) was modified as

$$
d=\frac{1}{2} \sum_{i=0}^{i_{\max }}\left|F_{\mathrm{PM}}\left(\varepsilon_{\mathrm{MC}, i}^{\mathrm{R}}\right)-F_{\mathrm{MC}}\left(\varepsilon_{\mathrm{MC}, i}^{\mathrm{R}}\right)\right| \Delta \varepsilon_{\mathrm{MC}},
$$

where $\Delta \varepsilon_{\mathrm{MC}}$ is the width of a sampling section for $\varepsilon$ in the $\mathrm{MC}$ and $\varepsilon_{\mathrm{MC}, i}^{\mathrm{R}}=\left(i+\frac{1}{2}\right) \Delta \varepsilon_{\mathrm{MC}}$ is the representative energy value of the section. $\Delta \varepsilon_{\mathrm{MC}}=1 \mathrm{eV}$ and $i_{\max }=99$ in the present sampling. The $d$ values are shown in the caption of figure 6 , and they are less than 0.01 .

Furthermore, logarithmic plots of $F(\varepsilon) / \sqrt{\varepsilon}$ are shown in figure 7 to confirm their decay in the high-energy tails. Statistical fluctuation is observed in $F_{\mathrm{MC}}(\varepsilon) / \sqrt{\varepsilon}$ at high $\varepsilon$, however, the PM results agree satisfactorily with the MC results. 


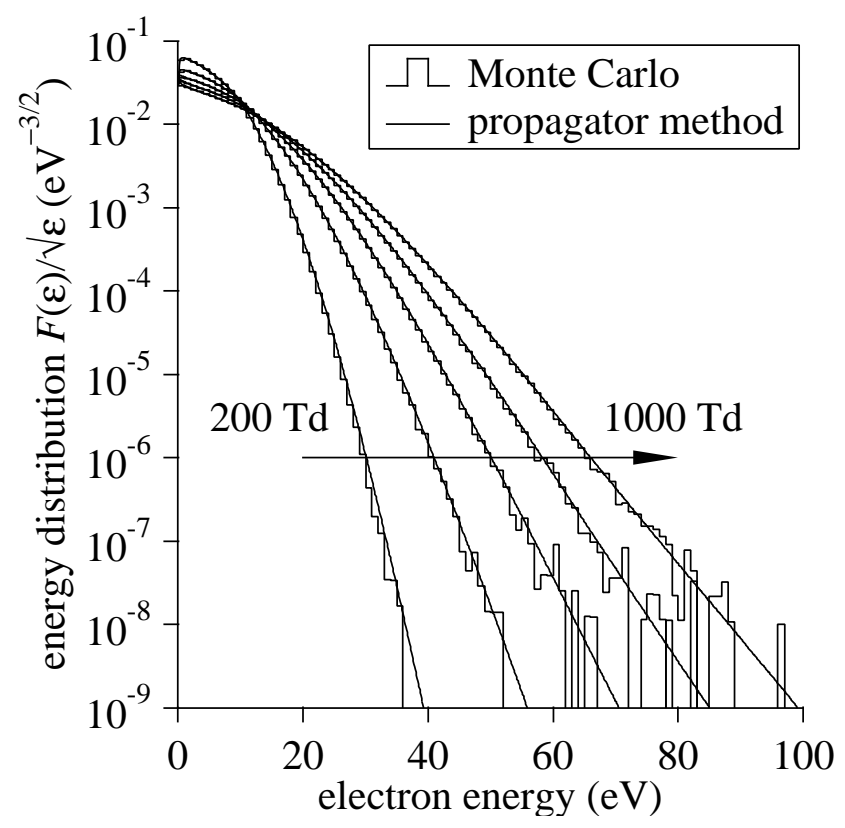

Figure 7. Electron energy distribution $F(\varepsilon) / \sqrt{\varepsilon}$ in $\mathrm{SF}_{6}$ replotted from figure 6 .

Figure 8 shows the $k$ th-order terms $F_{k}\left(\varepsilon, T_{\text {obs }}\right)$ of the Legendre polynomial expansion of the EEDF calculated by the PM and the MC at $1000 \mathrm{Td} . F_{0}(\varepsilon)$ and $F_{1}(\varepsilon)$ calculated by a BE analysis of two-term approximation are shown together. The EVDF was expanded as follows:

$$
\begin{aligned}
& 4 \pi v^{2} f(v, \theta, \phi, t)=\sum_{n=0}^{\infty} P_{k}(\cos \theta) f_{k}(v, t) \\
& f_{k}(v, t)=(2 k+1) \int_{\theta=0}^{\pi} \int_{\phi=0}^{2 \pi} P_{k}(\cos \theta) f(v, \theta, \phi, t) v^{2} \sin \theta \mathrm{d} \theta \mathrm{d} \phi .
\end{aligned}
$$

The $k$ th-order Legendre polynomial $P_{k}(x)$ is given as

$$
\begin{aligned}
& P_{0}(x)=1, \quad P_{1}(x)=x, \\
& P_{k}(x)=\frac{2 k-1}{k} x P_{k-1}(x)-\frac{k-1}{k} P_{k-2}(x) .
\end{aligned}
$$

The higher-order terms represent the anisotropy of the EVDF. $f_{k}(v, t)$ was converted into $F_{k}(\varepsilon, t)$ by the relation $F_{k}(\varepsilon, t) \mathrm{d} \varepsilon=f_{k}(v, t) \mathrm{d} v$, and their values are given as

$$
F_{k}\left(\varepsilon_{i}^{\mathrm{R}}, t\right)=\frac{2 k+1}{\Delta \varepsilon} \frac{\sum_{j} P_{k}\left(\cos \theta_{j}^{\mathrm{R}}\right) n_{i, j}(t)}{\sum_{i, j} n_{i, j}(t)} .
$$

The EEDF in figure 8 agrees well between the PM and the MC. Even a distorted EVDF under a high $E / N$ was calculated appropriately by the PM.

It should be noted here that the Legendre polynomial expansion was performed only to demonstrate the agreement with the results of the MC up to higher-order terms. This expansion is not necessary in the PM calculation itself. This is unlike the BE analyses that require the expansion of the EVDF to derive the simultaneous differential equations to be solved numerically. 


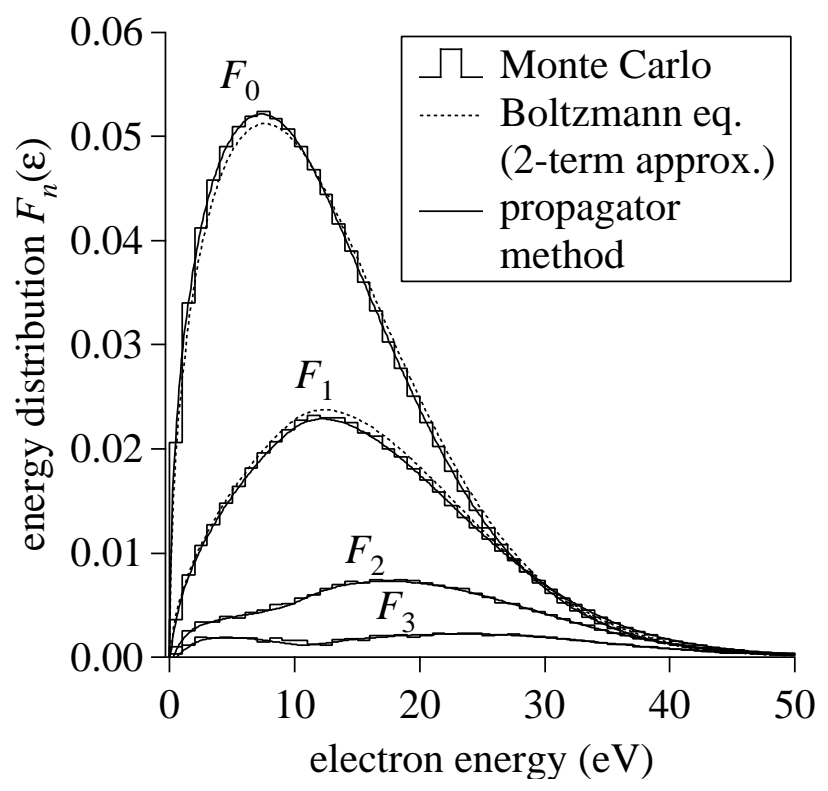

Figure 8. Electron energy distribution in $\mathrm{SF}_{6}$ at $E / N=1000 \mathrm{Td}(E=353.6 \mathrm{~V} / \mathrm{cm}$, $133 \mathrm{~Pa}$ at $273 \mathrm{~K}$ ): histograms, MC; broken curves, BE analysis (2-term approximation); and solid curves, PM. $F_{n}(\varepsilon)$ represents the $n$th order terms of Legendre polynomial expansion of the EEDF.

\subsection{Relaxation of swarm parameters}

Figure 9 shows the relaxation process of some swarm parameters. The results of the PM and the MC also agree with each other. A slight lead of the PM seen in the relaxation process indicates the influence of a numerical diffusion within cells. The electron transfer to a neighboring cell by acceleration would occur in the vicinity of the cell boundaries. Thus, the transferred electrons would stay near the boundary immediately after the transfer in a physical view. However, at the next time step, the electron distribution within a cell is treated to be uniform. This computational treatment causes the numerical diffusion and that induced a faster relaxation in the PM calculation.

Here, let us consider how to estimate the relaxation time, which is a factor to determine the computational load.

Figure 10 shows logarithmic plots of normalized time derivative $|\mathrm{d} X / \mathrm{d} t| /|X|$ for a parameter $X . X$ represents $\bar{\varepsilon}, W_{\mathrm{v}}, \nu_{\text {ion }}$ and $\nu_{\text {att }}$ calculated by the PM and $\bar{\varepsilon}$ and $W_{\mathrm{v}}$ by the MC. The MC results of $\nu_{\text {ion }}$ and $\nu_{\text {att }}$ are not shown here since they fluctuated significantly; the stochastic nature of the $\mathrm{MC}$ appeared strongly in the parameters directly based on collisions. The MC results of $\bar{\varepsilon}$ and $W_{\mathrm{v}}$ agree with those of the PM in an early period up to $1.0-1.5 \mathrm{~ns}$, but after that, the derivation of $|\mathrm{d} X / \mathrm{d} t| /|X|$ became difficult for a lack of stability due to statistical fluctuation.

The PM results, which are stable in contrast, indicate that the time derivative decays exponentially. At this time, it is inferred that $X$ itself has a form of 


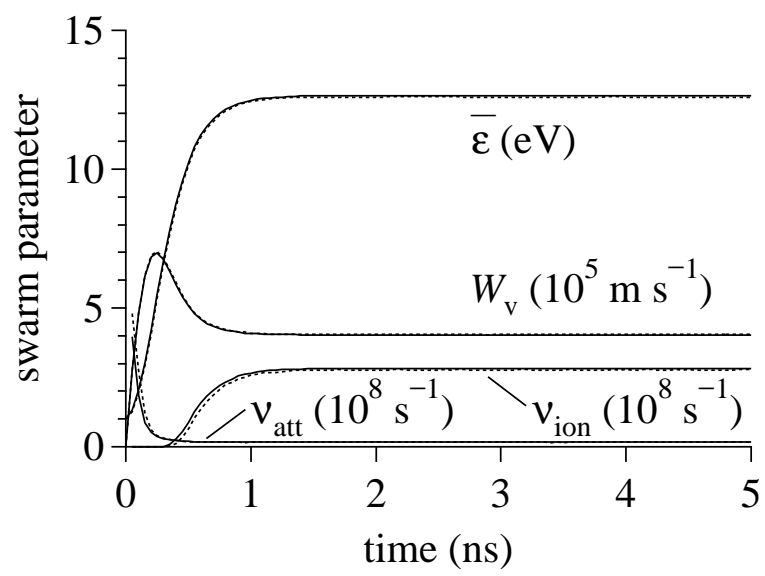

Figure 9. Physical relaxation process of mean electron energy $\bar{\varepsilon}$, drift velocity $W_{\mathrm{v}}$, ionization frequency $\nu_{\text {ion }}$ and attachment frequency $\nu_{\text {att }}$ in $\mathrm{SF}_{6}$ at $E / N=1000 \mathrm{Td}$ $(E=353.6 \mathrm{~V} / \mathrm{cm}, 133 \mathrm{~Pa}$ at $273 \mathrm{~K})$. Broken curves, MC; and solid curves, PM. The propagator operation was repeated $10^{5}$ times for the simulation of $5 \mathrm{~ns}$ with $\Delta t=0.05$ ps.

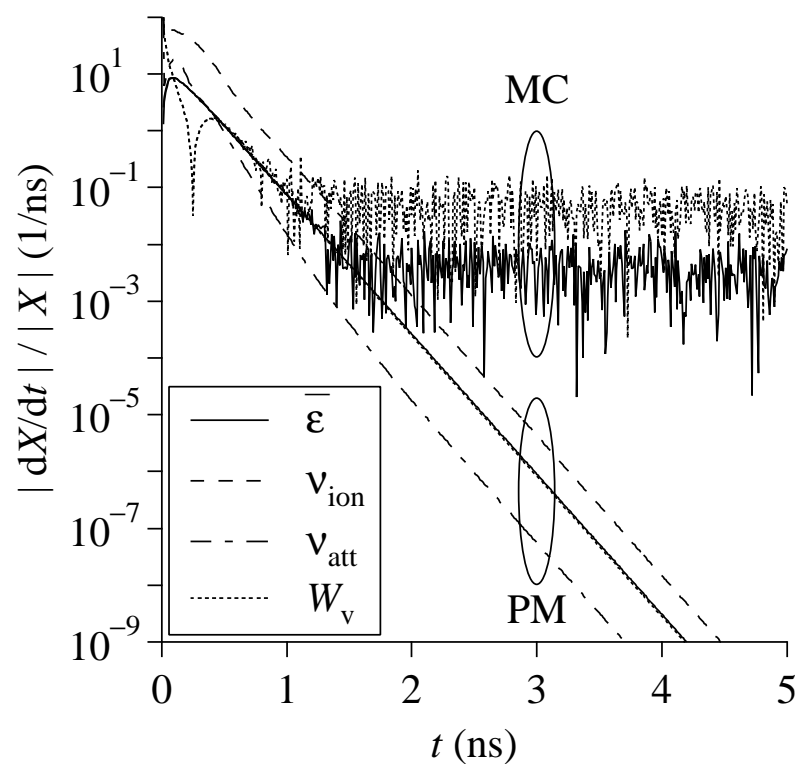

Figure 10. Decay of normalized time derivative $|\mathrm{d} X / \mathrm{d} t| /|X|$ of a parameter $X$. $X$ represents mean electron energy $\bar{\varepsilon}$, drift velocity $W_{\mathrm{v}}$, ionization frequency $\nu_{\text {ion }}$ and attachment frequency $\nu_{\text {att }}$ in $\mathrm{SF}_{6}$ at $E / N=1000 \mathrm{Td}(E=353.6 \mathrm{~V} / \mathrm{cm}, 133 \mathrm{~Pa}$ at $273 \mathrm{~K})$. The $\mathrm{MC}$ results of $\nu_{\mathrm{ion}}$ and $\nu_{\text {att }}$ are not shown because they fluctuated significantly.

$C_{1} \exp \left[C_{2} \exp \left(-t / \tau_{\text {conv }}\right)\right]$ in the convergence phase, where $C_{1}$ and $C_{2}$ are constants and $\tau_{\text {conv }}$ is the time constant of convergence $\left((\ln 10)^{-1}\right.$ digits per $\left.\tau_{\text {conv }}\right)$. The convergence of this type of function is slower than a simple exponential convergence of $C_{1}\left[1-\exp \left(-t / \tau_{\text {conv }}\right)\right]$. However, an important point is that the relaxation time in the 
Table 3. Time scales of relaxation and CPU times. The inverse of total collision frequency $\nu_{\text {total }}$, the momentum and energy relaxation times $\tau_{\mathrm{m}}$ and $\tau_{\mathrm{e}}$ in equations (37) and (38), respectively, the time constant $\tau_{\text {conv }}$ for the normalized time derivative of parameter, the five- and six-digit convergence times $T_{5}$ and $T_{6}$, and CPU times $T_{\mathrm{CPU}}^{\mathrm{PM}}$ and $T_{\mathrm{CPU}}^{\mathrm{MC}}$ for the calculation of electron swarm development in $\mathrm{SF}_{6}$ at $133 \mathrm{~Pa}$ at $273 \mathrm{~K}$ by the PM and MC for the observation period $T_{\mathrm{obs}}$. The cell resolution of the PM was $\varepsilon_{\max }=100 \mathrm{eV}, \Delta \varepsilon=0.1 \mathrm{eV}$ and $\Delta \theta=\pi / 180$. The number of electrons sampled in the $\mathrm{MC}$ was more than $10^{7}$ at $t=T_{\mathrm{obs}} \cdot T_{\mathrm{CPU}}^{\mathrm{PM}^{\prime}}$ is the CPU time of the PM calculation with a numerically accelerated relaxation scheme introduced in section 6 .

\begin{tabular}{|c|c|c|c|c|c|c|c|c|c|c|c|}
\hline $\begin{array}{l}E / N \\
(\mathrm{Td})\end{array}$ & $\begin{array}{c}\Delta t \\
(\mathrm{ps})\end{array}$ & $\begin{array}{l}T_{\text {obs }} \\
\text { (ns) }\end{array}$ & $\begin{array}{c}\nu_{\text {total }}^{-1} \\
(\mathrm{ps})\end{array}$ & $\begin{array}{c}\tau_{\mathrm{m}} \\
(\mathrm{ps})\end{array}$ & $\begin{array}{c}\tau_{\mathrm{e}} \\
(\mathrm{ns})\end{array}$ & $\begin{array}{c}\tau_{\text {conv }} \\
(\mathrm{ns})\end{array}$ & $\begin{array}{c}T_{5} \\
(\mathrm{~ns})\end{array}$ & $\begin{array}{c}T_{6} \\
\text { (ns) }\end{array}$ & $\begin{array}{c}T_{\mathrm{CPU}}^{\mathrm{MC}} \\
(\mathrm{s})\end{array}$ & $\begin{array}{c}T_{\mathrm{CPU}}^{\mathrm{PM}} \\
(\mathrm{s})\end{array}$ & $\begin{array}{c}T_{\mathrm{CPU}}^{\mathrm{PM}^{\prime}} \\
(\mathrm{s})\end{array}$ \\
\hline 200 & 0.05 & 20 & 114.3 & 01 & 5.92 & 1.731 & 19.67 & 19.97 & 33430 & 1068 & 0.560 \\
\hline 400 & 0.05 & 10 & 101.5 & 80.4 & 2.88 & 0.650 & 9.62 & 9.95 & 12760 & 451 & 0.328 \\
\hline 600 & 0.05 & 5 & 93.9 & 73.7 & 1.87 & 0.326 & 4.87 & 4.99 & 5739 & 222 & 0.212 \\
\hline 800 & 0.05 & 5 & 88.5 & 68.8 & 1.39 & 0.241 & 3.49 & 4.03 & 4768 & 224 & 0.164 \\
\hline 1000 & 0.05 & 5 & 84.2 & 64.9 & 1.12 & 0.177 & 2.56 & 2.97 & 3819 & 222 & 0.155 \\
\hline
\end{tabular}

convergence phase can be estimated with $\tau_{\text {conv }}$.

On the other hand, other measures of the momentum and energy relaxation times, $\tau_{\mathrm{m}}$ and $\tau_{\mathrm{e}}$, estimated after Makabe et al (1992) are given as

$$
\begin{aligned}
\tau_{\mathrm{m}} & =\frac{m W_{\mathrm{v}}}{e E}, \\
\tau_{\mathrm{e}} & =\left(2 \frac{m}{M} \nu_{\text {elastic }}+\frac{\sum_{k} \varepsilon_{\mathrm{ex}, k} \nu_{\mathrm{ex}, k}}{\bar{\varepsilon}}+\frac{\varepsilon_{\mathrm{ion}} \nu_{\mathrm{ion}}}{\bar{\varepsilon}}+\nu_{\mathrm{att}}\right)^{-1} .
\end{aligned}
$$

In the PM results, $\tau_{\mathrm{m}}$ was about $65-92 \mathrm{ps}$, and was comparable to the inverse of the total collision frequency, $\nu_{\text {total }}^{-1}$, i.e. the mean free time. $\tau_{\mathrm{e}}$ ranges from $1.1 \mathrm{~ns}$ at $1000 \mathrm{Td}$ to $5.9 \mathrm{~ns}$ at $200 \mathrm{Td}$. $\tau_{\mathrm{m}}$ seems less sensitive to $E / N$ than $\tau_{\mathrm{e}}$.

The $T_{\text {obs }}$ values chosen in the present benchmark are about 3-4 times as long as $\tau_{\mathrm{e}}$, within which the parameters achieved five- to six-digit convergence. The convergence times $T_{n}(n=5$ and 6$)$ were defined as such times that $\left|\bar{\nu}_{\text {ion }}(t)-\bar{\nu}_{\text {ion }}\left(T_{\text {obs }}\right)\right| /\left|\bar{\nu}_{\text {ion }}\left(T_{\text {obs }}\right)\right| \leq$ $10^{-n}$ for $t \geq T_{n}$, and were truncated into multiples of sampling time $T_{\text {sampling }}=0.01 \mathrm{~ns}$. These values of time scales are shown in table 3 together with results of computational times of the MC and the PM and $\tau_{\text {conv }}$ derived from the PM results.

It seems that $\tau_{\mathrm{e}}$ has a relatively good correlation with $T_{6}$ since $T_{6} / \tau_{\mathrm{e}}$ lies in a narrow range $2.7-3.5$, although $T_{6}$ includes an early relaxation phase of non-monotonous variation of parameters depending on the initial condition. It is not easy to estimate a sufficient relaxation time to achieve a required convergence level in prior to the simulation. However, a $T_{\text {obs }}$ value for the first trial simulation would be given as being several times of $\tau_{\mathrm{e}}$, and $\tau_{\mathrm{e}}$ could be estimated roughly from a Maxwellian EEDF, for example. After trial simulations, $T_{\text {obs }}$ would be adjusted referring to $\tau_{\text {conv }}$ derived from $|\mathrm{d} X / \mathrm{d} t| /|X|$, and justified by converged result finally. 


\section{Modification for numerically accelerated relaxation}

\subsection{Equilibrium solution}

When only the drift equilibrium solution is required, the relaxation process can be accelerated by a numerical method like the Gauss-Seidel method. Hereafter, we omit ' $(t)$ ' in the denotation of the quantities when their equilibrium values are referred to.

In drift equilibrium, the normalized EVDF is time-independent but the value of $f(\boldsymbol{v}, t)$ would show an exponential increase. $f(\boldsymbol{v}, t)$ satisfies

$$
\frac{\partial}{\partial t} f(\boldsymbol{v}, t)=\bar{\nu}_{\text {ion }} f(\boldsymbol{v}, t)=\left[-a \frac{\partial}{\partial v_{x}}+\left(\frac{\partial}{\partial t}\right)_{\text {coll }}\right] f(\boldsymbol{v}, t) .
$$

A discretized form of equation (39) is

$$
\begin{aligned}
\bar{\nu}_{\text {ion }} n_{i, j}(t)= & \left(\frac{\partial}{\partial t}\right)_{\text {acc }}^{\text {in }} n_{i, j}(t)-\left(\frac{\partial}{\partial t}\right)_{\text {acc }}^{\text {out }} n_{i, j}(t) \\
& +\left(\frac{\partial}{\partial t}\right)_{\text {coll }}^{\text {in }} n_{i, j}(t)-\left(\frac{\partial}{\partial t}\right)_{\text {coll }}^{\text {out }} n_{i, j}(t),
\end{aligned}
$$

where the superscripts and subscripts of the operators represent electron inflow and outflow due to acceleration and collisional events. The outflow terms, represented as the number of outflowing electrons per unit time here, are available from the descriptions in section 4. The inflows can also be given as the total number of electrons to be redistributed to $\mathrm{C}_{i, j}$ from such source cells $\mathrm{C}_{I, J}$ that $\mathrm{C}_{i, j}$ is one of the destination cells of $\mathrm{C}_{I, J}$.

The outflows can be calculated from $n_{i, j}(t)$ as

$$
\begin{aligned}
& \left(\frac{\partial}{\partial t}\right)_{\mathrm{acc}}^{\text {out }} n_{i, j}(t)=\frac{a S_{i, j}^{-\Delta \theta}+a S_{i, j}^{ \pm \Delta \varepsilon}}{V_{i, j}} n_{i, j}(t), \\
& \left(\frac{\partial}{\partial t}\right)_{\text {coll }}^{\text {out }} n_{i, j}(t)=\sum_{k} N q_{k}\left(v_{i}^{\mathrm{R}}\right) v_{i}^{\mathrm{R}} n_{i, j}(t) .
\end{aligned}
$$

One of the inflows, $(\partial / \partial t)_{\mathrm{acc}}^{\mathrm{in}} n_{i, j}(t)$, is the sum of the outflows from the two upstream neighbor cells of $\mathrm{C}_{i, j}$, which are $\mathrm{C}_{i, j+1}$ and $\mathrm{C}_{i \mp 1, j}\left(\mathrm{C}_{i-1, j}\right.$ for the forward cells and $\mathrm{C}_{i+1, j}$ for the backward cells):

$$
\left(\frac{\partial}{\partial t}\right)_{\mathrm{acc}}^{\mathrm{in}} n_{i, j}(t)=\frac{a S_{i \mp 1, j}^{ \pm \Delta \varepsilon}}{V_{i \mp 1, j}} n_{i \mp 1, j}(t)+\frac{a S_{i, j+1}^{-\Delta \theta}}{V_{i, j+1}} n_{i, j+1}(t) .
$$

$(\partial / \partial t)_{\text {coll }}^{\text {in }} n_{i, j}(t)$ involves the inflows due to all kinds of collisions:

$$
\left(\frac{\partial}{\partial t}\right)_{\text {coll }}^{\text {in }} n_{i, j}(t)=\left[\left(\frac{\partial}{\partial t}\right)_{\text {elastic }}^{\text {in }}+\left(\frac{\partial}{\partial t}\right)_{\text {ex }}^{\text {in }}+\left(\frac{\partial}{\partial t}\right)_{\text {ion }}^{\text {in }}\right] n_{i, j}(t),
$$

where there is no inflow concerned with electron attachment, i.e. $(\partial / \partial t)_{\mathrm{att}}^{\mathrm{in}} n_{i, j}(t)=0$. 
The source cells $\mathrm{C}_{I, J}$ supplying electrons to $\mathrm{C}_{i, j}$ by the collisional events are diverse. Those of elastic collision are of $I=i$ and all $J$. The corresponding inflow $(\partial / \partial t)_{\text {elastic }}^{\mathrm{in}} n_{i, j}$ is calculated as

$$
\left(\frac{\partial}{\partial t}\right)_{\text {elastic }}^{\text {in }} n_{i, j}(t)=\frac{\Omega_{j}}{4 \pi} \nu_{\text {elastic }, i} \sum_{J} n_{i, J}(t) .
$$

$\mathrm{C}_{I, J}$ of excitation collision are of $I=i+k_{\mathrm{ex}}$ and all $J$. The inflow $(\partial / \partial t)_{\mathrm{ex}}^{\mathrm{in}} n_{i, j}(t)$ is

$$
\left(\frac{\partial}{\partial t}\right)_{\mathrm{ex}}^{\mathrm{in}} n_{i, j}(t)=\frac{\Omega_{j}}{4 \pi} \nu_{\mathrm{ex}, i+k_{\mathrm{ex}}} \sum_{J} n_{i+k_{\mathrm{ex}}, J}(t) .
$$

When $i+k_{\text {ex }} \geq i_{\text {ceiling, }}$, the term is not calculated. When the gas has two or more excitation processes, this inflow calculation is made for each process. $\mathrm{C}_{I, J}$ of ionization collision are of $i+k_{\text {ion }} \leq I<i_{\text {ceiling }}$ and all $J$. The inflow $(\partial / \partial t)_{\text {ion }}^{\text {in }} n_{i, j}(t)$ is

$$
\left(\frac{\partial}{\partial t}\right)_{\text {ion }}^{\text {in }} n_{i, j}(t)=\frac{\Omega_{j}}{4 \pi} \sum_{I=i+k_{\text {ion }}}^{i_{\text {ceiling }}-1} \sum_{J} \delta_{\text {ion }}^{I \rightarrow i} \nu_{\text {ion }, I} n_{I, J}(t) .
$$

When two or more ionization processes are present, this term is calculated for all relevantly.

The outflows and inflows can be rearranged as

$$
\left[\bar{\nu}_{\text {ion }}+\left(\frac{\partial}{\partial t}\right)_{\text {acc }}^{\text {out }}+\left(\frac{\partial}{\partial t}\right)_{\text {coll }}^{\text {out }}\right] n_{i, j}(t)=\left(\frac{\partial}{\partial t}\right)_{\text {acc }}^{\text {in }} n_{i, j}(t)+\left(\frac{\partial}{\partial t}\right)_{\text {coll }}^{\text {in }} n_{i, j}(t) .(
$$

The terms in the left-hand side are proportional to $n_{i, j}(t)$. Those in the right-hand side are not directly calculated from $n_{i, j}(t)$ but from $n_{I, J}(t)$ of other source cells.

In equilibrium, equation (48) becomes

$$
n_{i, j}=\frac{(\partial / \partial t)_{\mathrm{coll}}^{\mathrm{in}} n_{i, j}+(\partial / \partial t)_{\mathrm{acc}}^{\mathrm{in}} n_{i, j}}{\bar{\nu}_{\mathrm{ion}}+a\left(S_{i, j}^{-\Delta \theta}+S_{i, j}^{ \pm \Delta \varepsilon}\right) / V_{i, j}+\sum_{k} N q_{k}\left(v_{i}^{\mathrm{R}}\right) v_{i}^{\mathrm{R}}} .
$$

This is the equation to be solved. Equation (49) is in practise simultaneous equations for $i_{\text {ceiling }} j_{\text {ceiling }}$ cells. $\Delta t$ is not cared in this calculation because it has already been cancelled between the numerator and the denominator. Thus, the restrictions of equations (26) and (27) on $\Delta t$ are not applied here.

\subsection{Numerical relaxation}

Under the same physical condition as in section 5 , starting from initial values of $n_{i, j}$, renewal of $n_{i, j}$ by equation (49) was repeated until the relative variations of $\bar{\varepsilon}$ and $\bar{\nu}_{\text {ion }}$ for one cycle of renewal became less than $10^{-7}$.

The renewal of $n_{i, j}$ was made from the backward (upstream) cells to the forward (downstream) cells so that the renewed values propagate to other cells immediately; i.e. from $\pi$ to $\frac{1}{2} \pi$ for $\theta$ and from $\varepsilon_{\max }$ to 0 for $\varepsilon$ for the backward cells; and from $\frac{1}{2} \pi$ to 0 for $\theta$ and from 0 to $\varepsilon_{\max }$ for $\varepsilon$ for the forward cells. This treatment is based on the idea of the Gauss-Seidel method. 
This renewal process in the PM is in part similar to the backward prolongation (Frost and Phelps 1962, Thomas 1969) to solve the BE in a form of differential equation with respect to $v$ or $\varepsilon$. In the backward prolongation, the $F(\varepsilon)$ values are renewed from the high- $\varepsilon$ end towards lower $\varepsilon$ since the BE includes terms referring to $F(\varepsilon)$ values at higher $\varepsilon$ for consideration of the electrons losing energies by inelastic collisions. On the other hand, the EVDF renewal in the PM proceeds firstly in the same direction for the backward cells as is in the backward prolongation, and next in the opposite direction for the forward cells. The number of electrons undergoing inelastic collisions at higher $\varepsilon$, involved in $(\partial / \partial t)_{\text {coll }}^{\text {in } n_{i, j}}$ in equation $(49)$, is to be calculated before the renewal process. Thus, this factor was treated to be unchanged during a cycle of the renewal. The renewed $n_{i, j}$ values of the backward cells are not immediately reflected to the renewal of the forward cells, and the idea of the Gauss-Seidel method was applied only to $(\partial / \partial t)_{\mathrm{acc}}^{\mathrm{in}} n_{i, j}$ in equation (49).

In the iteration of the relaxation of the BE, $\bar{\nu}_{\text {ion }}$ or the effective ionization coefficient $\bar{\alpha}_{\text {ion }}$ is often taken as the parameter to judge the convergence. Similar treatment with $\bar{\alpha}_{\text {ion }}$ was adopted in the PM calculation for the EEDF under a steady-state Townsend condition (Sugawara et al 1994). The present relaxation in the PM also adopted $\bar{\nu}_{\text {ion }}$ as one of the parameters for the convergence judgement.

Another difference of the present relaxation scheme in the PM from the GaussSeidel method was that the EVDF under numerical relaxation was not normalized yet. After the convergence of $\bar{\varepsilon}$ and $\bar{\nu}_{\text {ion }}$, the EVDF was normalized to satisfy $\sum_{i, j} n_{i, j}=1$.

\subsection{Results of numerical relaxation}

Figure 11 shows the relaxation process of the same swarm parameters as shown in figure 9. Their relative changes in an iteration step, i.e. $\left|X^{\prime}-X\right| /\left|X^{\prime}\right|$ for parameter values $X$ and $X^{\prime}$ before and after the renewal, respectively, are presented in figure 12 . The abscissas represent the iteration counts for substitution of renewed values for all $n_{i, j}$, thus the numerical relaxation no longer represents the elapse of physical time for the electron swarm. Figure 12 shows that the convergence of the differentials of parameters is exponential similarly to that of the time derivatives observed in the physical relaxation. The relaxation at $E / N=1000 \mathrm{Td}$ required only 52 cycles of renewal to achieve the seven-digit convergence. The result of the EEDF agreed well with that in figure 6 . The CPU time $T_{\mathrm{CPU}}^{\mathrm{PM}^{\prime}}$ required for the PM with the numerically accelerated relaxation scheme, shown in table 3 together with those for the original PM and the MC, was less than 1 s. Even in case of $E / N=200 \mathrm{Td}$, i.e. attachment-dominated case, the relaxation was completed with 220 cycles of renewal.

It seems that the convergence is more stable and faster in case $\bar{\nu}_{\text {ion }}>0$, i.e. ionization is dominant, perhaps because the significance of the deviation of the EVDF from the equilibrium solution is diluted by the increase of electrons. However, the guarantee of the convergence, e.g., in an extremely attachment-dominated case, is not confirmed yet. Applicable conditions for this numerical relaxation scheme is still under 


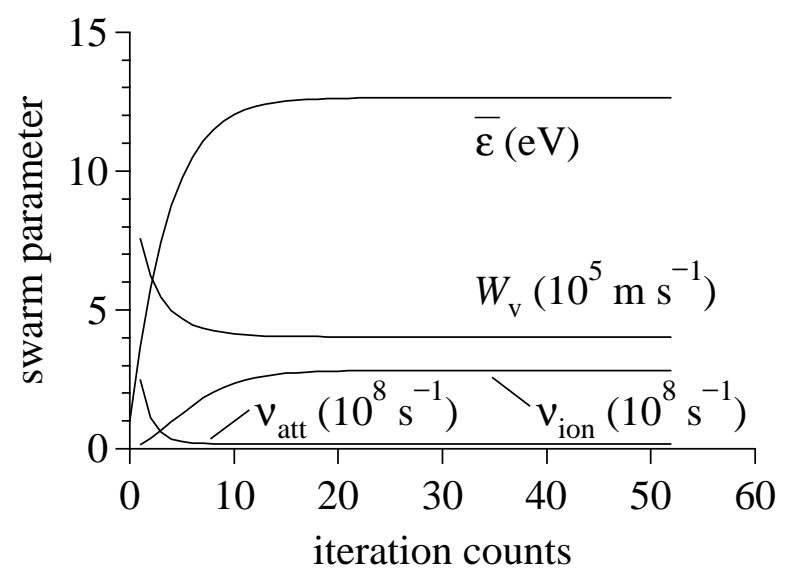

Figure 11. Numerical relaxation process of mean electron energy $\bar{\varepsilon}$, drift velocity $W_{\mathrm{v}}$, ionization frequency $\nu_{\text {ion }}$ and attachment frequency $\nu_{\text {att }}$ in $\mathrm{SF}_{6}$ at $E / N=1000 \mathrm{Td}$ $(E=353.6 \mathrm{~V} / \mathrm{cm}, 133 \mathrm{~Pa}$ at $273 \mathrm{~K})$ calculated by the $\mathrm{PM}$ with a numerically accelerated relaxation scheme. The propagator operation was repeated 52 times until reaching the equilibrium.

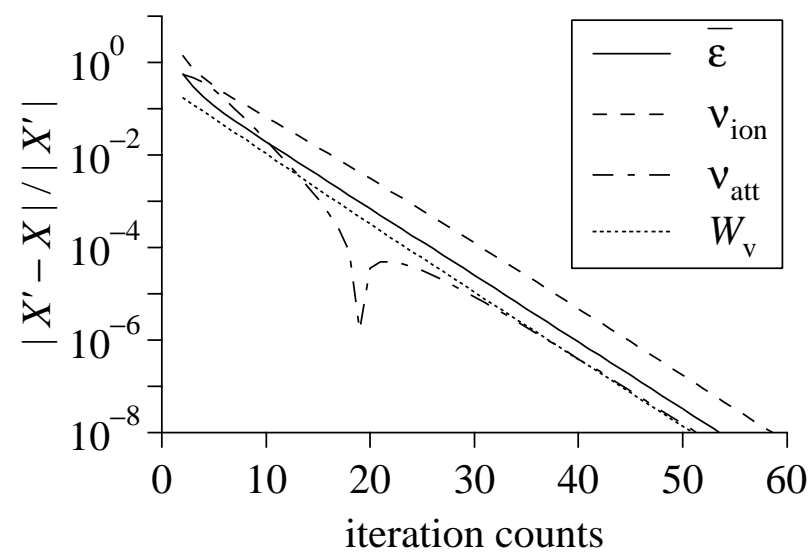

Figure 12. Decay of normalized differential $\left|X^{\prime}-X\right| /\left|X^{\prime}\right|$ for parameter values $X$ and $X^{\prime}$ before and after the renewal of $n_{i, j}$, respectively. $X$ represents mean electron energy $\bar{\varepsilon}$, drift velocity $W_{\mathrm{v}}$, ionization frequency $\nu_{\text {ion }}$ and attachment frequency $\nu_{\text {att }}$ in $\mathrm{SF}_{6}$ at $E / N=1000 \mathrm{Td}(E=353.6 \mathrm{~V} / \mathrm{cm}, 133 \mathrm{~Pa}$ at $273 \mathrm{~K})$ calculated by the $\mathrm{PM}$ with a numerically accelerated relaxation scheme.

investigation. 


\section{Extension for calculation of transport parameters used in fluid-model simulations}

\subsection{Parameters representing spatial development of electron swarms}

A fluid model simulation is often performed with the following one-dimensional electron continuity equation:

$$
\frac{\partial}{\partial t} f(x, t)=\bar{\nu}_{\text {ion }} f(x, t)-W_{\mathrm{r}} \frac{\partial}{\partial x} f(x, t)+D_{\mathrm{L}} \frac{\partial^{2}}{\partial x^{2}} f(x, t),
$$

where $f(x, t)=\int_{\boldsymbol{v}} \int_{y} \int_{z} f(x, y, z, \boldsymbol{v}, t) \mathrm{d} y \mathrm{~d} z \mathrm{~d} \boldsymbol{v}$ is the spatial electron distribution, $W_{\mathrm{r}}$ is the centroid drift velocity and $D_{\mathrm{L}}$ is the longitudinal diffusion coefficient. $\bar{\nu}_{\text {ion }}$ is derived from the EVDF using equation (15) for $\nu_{\text {ion }}$ and $\nu_{\text {att }}$. $W_{\mathrm{r}}$ and $D_{\mathrm{L}}$ are parameters defined on the basis of electron swarm development in real space as

$$
\begin{aligned}
& W_{\mathrm{r}}=\frac{\mathrm{d}}{\mathrm{d} t} G_{x}(t)=\frac{\mathrm{d}}{\mathrm{d} t} \frac{\int_{-\infty}^{\infty} x f(x, t) \mathrm{d} x}{\int_{-\infty}^{\infty} f(x, t) \mathrm{d} x}, \\
& D_{\mathrm{L}}=\frac{1}{2} \frac{\mathrm{d}}{\mathrm{d} t} \frac{\int_{-\infty}^{\infty}\left[x-G_{x}(t)\right]^{2} f(x, t) \mathrm{d} x}{\int_{-\infty}^{\infty} f(x, t) \mathrm{d} x},
\end{aligned}
$$

where $G_{x}(t)$ is the centroid position of the electron swarm in the $x$ direction.

$\bar{\nu}_{\text {ion }}, W_{\mathrm{r}}$ and $D_{\mathrm{L}}$, usually referred to as functions of $E$ or $\bar{\varepsilon}$ in the fluid-model simulations, are to be given by another self-consistent simulation method or as measured data. A way to derive them by the PM is presented in this section.

\subsection{Extension of the Boltzmann equation to moment equations}

Equations (51) and (52) represent that $W_{\mathrm{r}}$ and $D_{\mathrm{L}}$ are obtained from the first- and second-order moments, respectively. Let $m_{k}(\boldsymbol{v}, t)$ be the distribution function of the $k$ th order moment in velocity space. The moment equation for $m_{k}(\boldsymbol{v}, t)$ is obtained by integrating equation (1) with a weight of $x^{k}$ over $\boldsymbol{r}$ (Sugawara et al 1997, 1998):

$$
\begin{aligned}
m_{k}(\boldsymbol{v}, t) & =\int_{\boldsymbol{r}} x^{k} f(\boldsymbol{r}, \boldsymbol{v}, t) \mathrm{d} \boldsymbol{r} \\
\frac{\partial}{\partial t} m_{k}(\boldsymbol{v}, t) & =-a \frac{\partial}{\partial v_{x}} m_{k}(\boldsymbol{v}, t)+k v_{x} m_{k-1}(\boldsymbol{v}, t)+\left(\frac{\partial}{\partial t}\right)_{\text {coll }} m_{k}(\boldsymbol{v}, t) .
\end{aligned}
$$

$m_{0}(\boldsymbol{v}, t)$ is identical to $f(\boldsymbol{v}, t)$. The second term in the right-hand side of equation (54) appeared as the drift term representing the moment change due to the electron flight in real space. This term is missing for $k=0$, because the zeroth-order moment, which is the electron population, is unchanged in the flight.

$W_{\mathrm{r}}$ and $D_{\mathrm{L}}$ are obtained from $m_{k}(\boldsymbol{v}, t)$ as

$$
\begin{aligned}
M_{k}(t) & =\int_{\boldsymbol{v}} m_{k}(\boldsymbol{v}, t) \mathrm{d} \boldsymbol{v}, \\
W_{\mathrm{r}} & =\frac{\mathrm{d}}{\mathrm{d} t} \frac{M_{1}(t)}{M_{0}(t)}, \\
D_{\mathrm{L}} & =\frac{1}{2} \frac{\mathrm{d}}{\mathrm{d} t}\left[\frac{M_{2}(t)}{M_{0}(t)}-\left(\frac{M_{1}(t)}{M_{0}(t)}\right)^{2}\right],
\end{aligned}
$$


where $M_{k}(t)$ is the total amount of the $k$ th order moment in the electron swarm.

$W_{\mathrm{r}}$ and $D_{\mathrm{L}}$ defined in real space can be obtained by the PM calculation with cells defined in velocity space. Furthermore, even $f(x, t)$ can be composed using orthogonal bases of the Hermite polynomials with weights determined by $m_{k}(\boldsymbol{v}, t)$ of higher orders (Sugawara et al 1998). These are because the change of $x$ under a uniform $\boldsymbol{E}$ can be known from that of $\varepsilon$ or $v$ via a relation $\Delta \varepsilon=e E \Delta x$ and $v_{x}$ specified in each cell represents the change of $x$ per unit time.

\subsection{Temporal development of the moment distributions}

The first- and second-order moments within $\mathrm{C}_{i, j}, m_{1, i, j}(t)$ and $m_{2, i, j}(t)$, respectively, are introduced as new properties of $\mathrm{C}_{i, j}$ :

$$
m_{k, i, j}(t)=\int_{v=v_{i}}^{v_{i+1}} \int_{\theta=\theta_{j}}^{\theta_{j+1}} \int_{\phi=0}^{2 \pi} m_{k}(\boldsymbol{v}, t) v^{2} \sin \theta \mathrm{d} v \mathrm{~d} \theta \mathrm{d} \phi .
$$

One way to obtain the equilibrium values of $W_{\mathrm{r}}$ and $D_{\mathrm{L}}$ is to calculate the temporal variations of $m_{1, i, j}(t)$ and $m_{2, i, j}(t)$ by equation (54) simultaneously with that for $m_{0, i, j}(t)$. The initial condition may be, e.g., a Maxwellian for $m_{0, i, j}(0)$ as in equation (30), $m_{1, i, j}(0)=0$ and $m_{2, i, j}(0)=0$. In addition to the intercellular transfer of the number of electrons, those of the $k$ th-order moments are operated by the same acceleration and collision propagators. Because the moments accompany the electron transfer and thus their variations are proportional to the concerning electron population, the propagators are common for $m_{k, i, j}(t)$. In addition, the effect of the drift term in equation (54) is to be taken into account relevantly.

The temporal variations of $W_{\mathrm{r}}$ and $D_{\mathrm{L}}$ available from equations (56) and (57) are physical relaxation process. The convergence of them can be judged in the same way as done for the EVDF. Their relaxation processes in $\mathrm{CF}_{4}$ were presented in Sugawara and Sakai (2006) together with those of $D_{k}$ up to $k=6$, thus they are not repeated here. Instead, their numerical relaxation is performed in the next subsection as a new effort.

\subsection{Numerical relaxation scheme for higher-order moments}

As done for the EVDF in section 6, the numerical relaxation scheme is applicable to the moment distributions. Instead of $m_{k}(\boldsymbol{v}, t)$ defined in laboratory system, we calculate $m_{k}^{\mathrm{C}}(\boldsymbol{v}, t)$, which is the distribution of the $k$ th-order moment defined in centroid system relative to $G_{x}(t)$ :

$$
\begin{aligned}
m_{0}^{\mathrm{C}}(\boldsymbol{v}, t) & =\int_{\boldsymbol{r}} f(\boldsymbol{r}, \boldsymbol{v}, t) \mathrm{d} \boldsymbol{r}=m_{0}(\boldsymbol{v}, t), \\
m_{1}^{\mathrm{C}}(\boldsymbol{v}, t) & =\int_{\boldsymbol{r}}\left[x-G_{x}(t)\right] f(\boldsymbol{r}, \boldsymbol{v}, t) \mathrm{d} \boldsymbol{r}=m_{1}(\boldsymbol{v}, t)-G_{x}(t) m_{0}(\boldsymbol{v}, t), \\
m_{2}^{\mathrm{C}}(\boldsymbol{v}, t) & =\int_{\boldsymbol{r}}\left[x-G_{x}(t)\right]^{2} f(\boldsymbol{r}, \boldsymbol{v}, t) \mathrm{d} \boldsymbol{r} \\
& =m_{2}(\boldsymbol{v}, t)-2 G_{x}(t) m_{1}(\boldsymbol{v}, t)+\left[G_{x}(t)\right]^{2} m_{0}(\boldsymbol{v}, t), \\
m_{k, i, j}^{\mathrm{C}}(t) & =\int_{v=v_{i}}^{v_{i+1}} \int_{\theta=\theta_{j}}^{\theta_{j+1}} \int_{\phi=0}^{2 \pi} m_{k}^{\mathrm{C}}(\boldsymbol{v}, t) v^{2} \sin \theta \mathrm{d} v \mathrm{~d} \theta \mathrm{d} \phi .
\end{aligned}
$$


The total amount $M_{k}^{\mathrm{C}}(t)$ of the $k$ th-order moment in centroid system is

$$
\begin{aligned}
& M_{0}^{\mathrm{C}}(t)=\int_{\boldsymbol{v}} m_{0}^{\mathrm{C}}(\boldsymbol{v}, t) \mathrm{d} \boldsymbol{v}=M_{0}(t), \\
& M_{1}^{\mathrm{C}}(t)=\int_{\boldsymbol{v}} m_{1}^{\mathrm{C}}(\boldsymbol{v}, t) \mathrm{d} \boldsymbol{v}=M_{1}(t)-G_{x}(t) M_{0}(t)=0, \\
& M_{2}^{\mathrm{C}}(t)=\int_{\boldsymbol{v}} m_{2}^{\mathrm{C}}(\boldsymbol{v}, t) \mathrm{d} \boldsymbol{v}=M_{2}(t)-\frac{\left[M_{1}(t)\right]^{2}}{M_{0}(t)} .
\end{aligned}
$$

By substituting the relation between $m_{1}(\boldsymbol{v}, t)$ and $m_{1}^{\mathrm{C}}(\boldsymbol{v}, t)$ in equation (60) into equation (54) for $k=1$, we obtain

$$
\begin{aligned}
\bar{\nu}_{\text {ion }} & {\left[m_{1}^{\mathrm{C}}(\boldsymbol{v}, t)+G_{x}(t) m_{0}^{\mathrm{C}}(\boldsymbol{v}, t)\right]+W_{\mathrm{r}} m_{0}^{\mathrm{C}}(\boldsymbol{v}, t) } \\
= & -a \frac{\partial}{\partial v_{x}}\left[m_{1}^{\mathrm{C}}(\boldsymbol{v}, t)+G_{x}(t) m_{0}^{\mathrm{C}}(\boldsymbol{v}, t)\right]+v_{x} m_{0}^{\mathrm{C}}(\boldsymbol{v}, t) \\
& +\left(\frac{\partial}{\partial t}\right)_{\text {coll }}\left[m_{1}^{\mathrm{C}}(\boldsymbol{v}, t)+G_{x}(t) m_{0}^{\mathrm{C}}(\boldsymbol{v}, t)\right] .
\end{aligned}
$$

The terms including $G_{x}(t)$ vanish for the relation in equation (39), then

$$
\begin{aligned}
\bar{\nu}_{\text {ion }} m_{1}^{\mathrm{C}}(\boldsymbol{v}, t)= & -a \frac{\partial}{\partial v_{x}} m_{1}^{\mathrm{C}}(\boldsymbol{v}, t)+\left(\frac{\partial}{\partial t}\right)_{\text {coll }} m_{1}^{\mathrm{C}}(\boldsymbol{v}, t) \\
& +\left(v_{x}-W_{\mathrm{r}}\right) m_{0}^{\mathrm{C}}(\boldsymbol{v}, t) .
\end{aligned}
$$

The discretization of this equation for $m_{k, i, j}^{\mathrm{C}}(t)$ and the separation of the inflow and outflow due to the acceleration and collisional events as done in equation (40) give the following equation to be solved:

$$
m_{1, i, j}^{\mathrm{C}}=\frac{(\partial / \partial t)_{\mathrm{coll}}^{\mathrm{in}} m_{1, i, j}^{\mathrm{C}}+(\partial / \partial t)_{\mathrm{acc}}^{\mathrm{in}} m_{1, i, j}^{\mathrm{C}}+\left(v_{i}^{\mathrm{R}} \cos \theta_{j}^{\mathrm{R}}-W_{\mathrm{r}}\right) m_{0, i, j}^{\mathrm{C}}}{\bar{\nu}_{\text {ion }}+a\left(S_{i, j}^{-\Delta \theta}+S_{i, j}^{ \pm \Delta \varepsilon}\right) / V_{i, j}+\sum_{k} N q_{k}\left(v_{i}^{\mathrm{R}}\right) v_{i}^{\mathrm{R}}} .
$$

This equation corresponds to equation (49) for $n_{i, j}=m_{0, i, j}^{\mathrm{C}}$. By similar substitution of equation (61) into $m_{2}(\boldsymbol{v}, t)$ in equation (54) for $k=2$ and rearrangement of the terms, we obtain

$$
m_{2, i, j}^{\mathrm{C}}=\frac{(\partial / \partial t)_{\text {coll }}^{\mathrm{in}} m_{2, i, j}^{\mathrm{C}}+(\partial / \partial t)_{\mathrm{acc}}^{\mathrm{in}} m_{2, i, j}^{\mathrm{C}}+2\left(v_{i}^{\mathrm{R}} \cos \theta_{j}^{\mathrm{R}}-W_{\mathrm{r}}\right) m_{1, i, j}^{\mathrm{C}}}{\bar{\nu}_{\mathrm{ion}}+a\left(S_{i, j}^{-\Delta \theta}+S_{i, j}^{ \pm \Delta}\right) / V_{i, j}+\sum_{k} N q_{k}\left(v_{i}^{\mathrm{R}}\right) v_{i}^{\mathrm{R}}} .
$$

Using $m_{k}^{\mathrm{C}}(\boldsymbol{v}, t), W_{\mathrm{r}}$ and $D_{\mathrm{L}}$ are rewritten as

$$
\begin{aligned}
W_{\mathrm{r}}= & \frac{\int_{\boldsymbol{v}} v_{x} m_{0}^{\mathrm{C}}(\boldsymbol{v}, t) \mathrm{d} \boldsymbol{v}}{\int_{\boldsymbol{v}} m_{0}^{\mathrm{C}}(\boldsymbol{v}, t) \mathrm{d} \boldsymbol{v}}+\frac{\int_{\boldsymbol{v}}\left[N q_{\text {ion }}(v) v-N q_{\mathrm{att}}(v) v\right] m_{1}^{\mathrm{C}}(\boldsymbol{v}, t) \mathrm{d} \boldsymbol{v}}{\int_{\boldsymbol{v}} m_{0}^{\mathrm{C}}(\boldsymbol{v}, t) \mathrm{d} \boldsymbol{v}} \\
= & \frac{\sum_{i, j} v_{i}^{\mathrm{R}} \cos \theta_{j}^{\mathrm{R}} m_{0, i, j}^{\mathrm{C}}}{\sum_{i, j} m_{0, i, j}^{\mathrm{C}}}+\frac{\sum_{i, j}\left[N q_{\text {ion }}\left(v_{i}^{\mathrm{R}}\right) v_{i}^{\mathrm{R}}-N q_{\mathrm{att}}\left(v_{i}^{\mathrm{R}}\right) v_{i}^{\mathrm{R}}\right] m_{1, i, j}^{\mathrm{C}}}{\sum_{i, j} m_{0, i, j}^{\mathrm{C}}} \\
D_{\mathrm{L}}= & \frac{\int_{\boldsymbol{v}}\left[v_{x}-W_{\mathrm{r}}(t)\right] m_{1}^{\mathrm{C}}(\boldsymbol{v}, t) \mathrm{d} \boldsymbol{v}}{\int_{\boldsymbol{v}} m_{0}^{\mathrm{C}}(\boldsymbol{v}, t) \mathrm{d} \boldsymbol{v}} \\
& +\frac{1}{2} \frac{\int_{\boldsymbol{v}}\left[N q_{\text {ion }}(v) v-N q_{\mathrm{att}}(v) v-\bar{\nu}_{\text {ion }}(t)\right] m_{2}^{\mathrm{C}}(\boldsymbol{v}, t) \mathrm{d} \boldsymbol{v}}{\int_{\boldsymbol{v}} m_{0}^{\mathrm{C}}(\boldsymbol{v}, t) \mathrm{d} \boldsymbol{v}} \\
= & \frac{\sum_{i, j}\left(v_{i}^{\mathrm{R}} \cos \theta_{j}^{\mathrm{R}}-W_{\mathrm{r}}\right) m_{1, i, j}^{\mathrm{C}}}{\sum_{i, j} m_{0, i, j}^{\mathrm{C}}}
\end{aligned}
$$




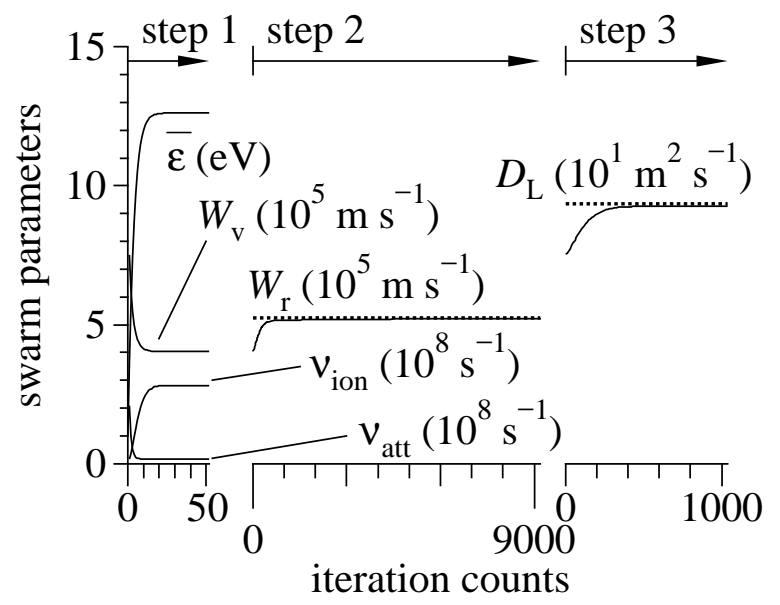

Figure 13. Numerical relaxation processes of centroid drift velocity $W_{\mathrm{r}}$ and longitudinal diffusion coefficient $D_{\mathrm{L}}$ calculated by the PM with a numerically accelerated relaxation scheme after the relaxation process shown in figure 11. $\mathrm{SF}_{6}$ at $E / N=1000 \mathrm{Td}(E=353.6 \mathrm{~V} / \mathrm{cm}, 133 \mathrm{~Pa}$ at $273 \mathrm{~K})$. The broken lines represent the equilibrium values of $W_{\mathrm{r}}$ and $D_{\mathrm{L}}$ obtained by the MC calculated for figure 9 .

$$
+\frac{1}{2} \frac{\sum_{i, j}\left[N q_{\text {ion }}\left(v_{i}^{\mathrm{R}}\right) v_{i}^{\mathrm{R}}-N q_{\mathrm{att}}\left(v_{i}^{\mathrm{R}}\right) v_{i}^{\mathrm{R}}-\bar{\nu}_{\text {ion }}\right] m_{2, i, j}^{\mathrm{C}}}{\sum_{i, j} m_{0, i, j}^{\mathrm{C}}} .
$$

The second term in the right-hand side of equation (70) represents that off-centroid electron generation and loss induce a centroid shift within the electron swarm (Tagashira et al 1977, Sugawara et al 1997). That of equation (71) similarly represents that the effect of off-average electron population change dependent on $v$ is added to the first term representing the basic diffusion due to electron motion.

Equation (54) represents that the developments of $m_{1}(\boldsymbol{v}, t)$ and $m_{2}(\boldsymbol{v}, t)$, thus $m_{1}^{\mathrm{C}}(\boldsymbol{v}, t)$ and $m_{2}^{\mathrm{C}}(\boldsymbol{v}, t)$ as well, are dependent on the lower-order moments while the calculation of $m_{0}(\boldsymbol{v}, t)$ is standing alone. Then, the numerical relaxation is carried out step by step. First, we obtain the normalized equilibrium solution of $m_{0}^{\mathrm{C}}(\boldsymbol{v})$. Next, the relaxation scheme is applied to $m_{1}^{\mathrm{C}}(\boldsymbol{v})$ under the fixed $m_{0}^{\mathrm{C}}(\boldsymbol{v})$ solution. The last step is the relaxation of $m_{2}^{\mathrm{C}}(\boldsymbol{v})$ under the solutions of $m_{0}^{\mathrm{C}}(\boldsymbol{v})$ and $m_{1}^{\mathrm{C}}(\boldsymbol{v})$. The renewals of $m_{1, i, j}^{\mathrm{C}}$ and $m_{2, i, j}^{\mathrm{C}}$ are made in the same order for $i$ and $j$ as was done for $n_{i, j}$ in section 6.2.

Figure 13 shows a result of this three-step relaxation in $\mathrm{SF}_{6}$ at $E / N=1000 \mathrm{Td}$. Step 1 is common with figure 11. The relaxations of $W_{\mathrm{r}}$ and $D_{\mathrm{L}}$ were terminated when the relative variation of the parameter for one cycle of renewal became less than $10^{-7}$ in the same way as in section 6 . Their converged values are listed in table 4 together with the CPU time $T_{\mathrm{CPU}}^{\mathrm{PM}^{\prime \prime}}$ and the iteration counts for the three-step relaxation at $E / N=200$ $1000 \mathrm{Td}$. The PM calculation for $W_{\mathrm{r}}$ and $D_{\mathrm{L}}$ with the numerically accelerated relaxation scheme reproduced the $W_{\mathrm{r}}$ and $D_{\mathrm{L}}$ values with discrepancies up to a few percents, except for the case of $\bar{\nu}_{\text {ion }}$ near zero at $400 \mathrm{Td}$. 
Table 4. Electron transport parameters in $\mathrm{SF}_{6}$ at $133 \mathrm{~Pa}$ at $273 \mathrm{~K}$ calculated by the Monte Carlo (MC) simulation and the propagator method (PM) with three-step numerical relaxation, and iteration counts needed for convergence of seven digits in the PM.

\begin{tabular}{|c|c|c|c|c|c|}
\hline \multirow[t]{3}{*}{$E / N(\mathrm{Td})$} & method & $\bar{\nu}_{\text {ion }}\left(\mathrm{s}^{-1}\right)$ & $\bar{\varepsilon}(\mathrm{eV})$ & $W_{\mathrm{r}}\left(\mathrm{m} \mathrm{s}^{-1}\right)$ & $D_{\mathrm{L}}\left(\mathrm{m}^{2} \mathrm{~s}^{-1}\right)$ \\
\hline & & \multicolumn{4}{|c|}{ relative discrepancy between $\mathrm{MC}$ and $\mathrm{PM}$} \\
\hline & $T_{\mathrm{CPU}}^{\mathrm{PM}^{\prime \prime}}(\mathrm{s})$ & \multicolumn{4}{|c|}{ iteration counts in the PM calculation } \\
\hline \multirow[t]{4}{*}{200} & $\mathrm{MC}$ & $-2.245 \times 10^{7}$ & 6.602 & $1.304 \times 10^{5}$ & 61.28 \\
\hline & $\mathrm{PM}$ & $-2.288 \times 10^{7}$ & 6.676 & $1.291 \times 10^{5}$ & 63.33 \\
\hline & & $1.92 \%$ & $1.12 \%$ & $1.00 \%$ & $3.35 \%$ \\
\hline & 5 & 219 & & 1097 & 1114 \\
\hline \multirow[t]{4}{*}{400} & $\mathrm{MC}$ & $8.412 \times 10^{6}$ & 8.522 & $2.345 \times 10^{5}$ & 76.30 \\
\hline & PM & $9.358 \times 10^{6}$ & 8.576 & $2.343 \times 10^{5}$ & 77.15 \\
\hline & & $11.25 \%$ & $0.63 \%$ & $0.09 \%$ & $1.11 \%$ \\
\hline & 4 & 127 & & 1235 & 479 \\
\hline \multirow[t]{4}{*}{600} & $\mathrm{MC}$ & $7.030 \times 10^{7}$ & 10.06 & $3.384 \times 10^{5}$ & 85.74 \\
\hline & PM & $7.236 \times 10^{7}$ & 10.10 & $3.374 \times 10^{5}$ & 86.44 \\
\hline & & $2.93 \%$ & $0.40 \%$ & $0.30 \%$ & $0.42 \%$ \\
\hline & 2 & 81 & & 305 & 387 \\
\hline \multirow[t]{4}{*}{800} & $\mathrm{MC}$ & $1.566 \times 10^{8}$ & 11.39 & $4.351 \times 10^{5}$ & 90.42 \\
\hline & $\mathrm{PM}$ & $1.611 \times 10^{8}$ & 11.43 & $4.340 \times 10^{5}$ & 90.80 \\
\hline & & $2.87 \%$ & $0.35 \%$ & $0.25 \%$ & $0.42 \%$ \\
\hline & 1 & 62 & & 351 & 275 \\
\hline \multirow[t]{4}{*}{1000} & $\mathrm{MC}$ & $2.622 \times 10^{8}$ & 12.60 & $5.237 \times 10^{5}$ & 93.48 \\
\hline & $\mathrm{PM}$ & $2.650 \times 10^{8}$ & 12.63 & $5.220 \times 10^{5}$ & 92.81 \\
\hline & & $1.07 \%$ & $0.24 \%$ & $0.32 \%$ & $0.72 \%$ \\
\hline & 25 & 52 & & 9210 & 1040 \\
\hline
\end{tabular}




\section{Discussion}

\subsection{Characteristics of the propagator method}

The cells in the present PM are concentric around $\boldsymbol{v}=0$ and their division is uniform with respect to $\varepsilon$. This configuration is convenient for the treatments of isotropic electron scattering and the energy conservation in the inelastic collisions. The dependence of the accuracy of the PM calculation on the cell resolution is a point to be investigated in future for reliable derivation of the electron swarm parameters.

Treatment of the electron acceleration as the electron outflow through the downstream cell boundaries keeps the mathematical aspect of the electron motion in the BE unimpaired. The intercellular electron transfer by the acceleration is limited for those between neighboring cells, that keeps the intercellular relationship simple. If the destination cells of the electron transfer are chosen on the basis of the arrival position of the Lagrangian cell (moving cell) departing from the source cell, the calculation of the overlapping volumes between the Lagrangian cell and the destination cells to determine the redistribution ratios for the electron transfer would be more complicated.

By operating the number of electrons as a primary property of the cells in the calculation of the temporal development of electron swarms, the PM calculation can guarantee the particle conservation. As a result, the PM calculation is stable although it is open-ended.

A similarity between the PM and a particle-in-cell MC simulation is often mentioned from the viewpoint of the intercellular particle transfer. However, one of the most significant differences is that the PM calculation is based on the expected values for the stochastic processes. It is an advantage of the PM that the PM is free from statistical fluctuation. Furthermore, since the properties of the cells, such as the electron population and the amount of moment, are stored in arrays in the PM calculation and most of the calculation steps do not require judgement for branching, a considerable part of the PM calculation will be parallelized when vector processing is available in the computational facility.

\subsection{Numerical relaxation scheme for the propagator method}

The numerical relaxation scheme like the Gauss-Seidel method was applicable not only to the EVDF but also to the higher-order moment distributions. However, the dependence of the convergence speed and the stability on the resolution of the cells and physical conditions have never been clarified yet. Empirically, some tendencies have been observed. For example, in some cases, the convergence of $m_{1}^{\mathrm{C}}(\boldsymbol{v})$ for $W_{\mathrm{r}}$, which is an odd-order parameter, took more iteration than those of $m_{0}^{\mathrm{C}}(\boldsymbol{v})$ and $m_{2}^{\mathrm{C}}(\boldsymbol{v})$ of even orders for $\bar{\nu}_{\text {ion }}$ and $D_{\mathrm{L}}$, respectively. The convergence of the EVDF becomes slower when $\bar{\nu}_{\text {ion }}$ decreases. Quantitative analysis for these tendencies is left for further investigation.

The normalized solutions of $m_{0}^{\mathrm{C}}(\boldsymbol{v})$ and $m_{1}^{\mathrm{C}}(\boldsymbol{v})$ seem to be unique. On the other hand, that of $m_{2}^{\mathrm{C}}(\boldsymbol{v})$ seems to be arbitrary even in equilibrium, because a constant $D_{\mathrm{L}}$ 
in equilibrium implies that the variance $[\sigma(t)]^{2}=M_{2}^{\mathrm{C}}(t) / M_{0}^{\mathrm{C}}(t)$ of the electron swarm increases linearly with $t$ under an exponential growth of $M_{0}^{\mathrm{C}}(t)$. A possible modification to make the solution of $m_{2}^{\mathrm{C}}(\boldsymbol{v})$ unique would be further normalization of $m_{2}^{\mathrm{C}}(\boldsymbol{v})$ by the standard deviation $\sigma(t)$ assuming $\sigma(t) \propto \sqrt{t}$. It was demonstrated that the normalized shape of $f(x, t)$ tends to a Gaussian (Sugawara et al 1998) and $\sigma(t)$ was used as a measure to normalize its shape. This issue is also left for future improvement of the relaxation technique of the PM together with interest in the stability of the convergence and its mathematical guarantee.

\subsection{Further extension of the propagator method}

A possible trial would be application of the numerical relaxation scheme to derivation of the transverse diffusion coefficient $D_{\mathrm{T}}$. Two-dimensional fluid simulations would demand direction-dependent diffusion coefficient data. The PM can derive $D_{\mathrm{T}}$ with simultaneous moment equations for the transverse moments weighted by $y$ and $y^{2}$ (Sugawara et al 1999), where $y$ is the position in a direction perpendicular to $\boldsymbol{E}$. This calculation requires three more quantities as new properties of $\mathrm{C}_{i, j}$ : one for the component of the first-order $y$ moment and two for the sub-components of the secondorder $y$ moment.

Temporally varying $\boldsymbol{E}$ was considered for radio-frequency and impulse electric fields as mentioned in section 1. Spatially non-uniform $\boldsymbol{E}$ was also treated by Sommerer et al (1989) in a parallel-plane electrodes model. These kinds of non-uniformity would have wide applications in practical problems.

Extension of the dimension of the variable space would require a huge memory storage. A one-dimensional parallel-plane electrodes model needs three-variable phase space $(x, v, \theta)$. A system in a spherical symmetry also needs three-variable phase space $\left(r, v_{\|}, v_{\perp}\right)$. Up-to-date computers can allocate three-dimensional arrays for the cells with a sufficient resolutions for $x, v$ and $\theta$. On the other hand, an axially symmetric threedimensional cylindrical model requires five-variable phase space $\left(r, z, v_{r}, v_{z}, v_{\theta}\right)$. The cell divisions for all of the five variables and their calculation would be a heavy load even though it might be possible for large-scale mainframe machines.

Another possibility is consideration of uniform and static magnetic field $\boldsymbol{B}$ crossed with $\boldsymbol{E}$ at angle $\alpha$. Three-variable velocity space, e.g., $(v, \theta, \phi)$ is required to calculate the EVDF under $\boldsymbol{E} \times \boldsymbol{B}$ fields. A complexity of this system is that the electron acceleration by the Lorentz force is velocity-dependent. The electron flow in velocity space becomes rotational (when $\alpha=\frac{1}{2} \pi$ ) or helical (when $\alpha \neq \frac{1}{2} \pi$ ) around an axis characterized by the $\boldsymbol{E} \times \boldsymbol{B}$ drift velocity $(E / B) \sin \alpha$. It is necessary to express such an electron flow by the acceleration propagator. 


\section{Conclusions}

The calculation scheme of the PM, which is a numerical approach to solve the BE for the EVDF and electron transport parameters under dc electric fields, was described in detail. The design of the cells in velocity space and the treatment of the intercellular electron transfer expressed as the acceleration and collision propagators were illustrated.

The PM calculation of the electron swarm development along physical time elapse was stable. The relaxation process of some swarm parameters and the equilibrium EEDF calculated by the PM showed fine agreement with those obtained by a MC simulation.

A numerical relaxation scheme like the Gauss-Seidel method was newly applied to the calculation of the EVDF in drift equilibrium. The iteration and the computational time required for the convergence were drastically reduced by this scheme down to the order of magnitude of about 1/1000 of the conventional relaxation along physical time elapse. Although this ratio is dependent on the choice of the angle and energy resolutions, the time step, and the physical relaxation time, the accelerated relaxation scheme is beneficial to the PM calculation. This scheme was also applied to the firstand second-order moment equations derived from the BE to obtain $W_{\mathrm{r}}$ and $D_{\mathrm{L}}$. The convergence of the higher-order moment distributions took longer time than that of the EVDF, but completed in a shorter time than for the procedure tracing the physical relaxation.

The PM is a calculation scheme requiring a huge memory capacity. However, the PM has attractive advantages such as stability in calculation of temporal development of electron swarms, freedom from statistical fluctuation, possibility of parallel computing, and so on. In the modern enriched computational environment, further extensions of the PM for higher dimension of variable space, higher orders of moments, spatiotemporal non-uniformity of the fields, and consideration of magnetic fields are expected as challenging efforts.

\section{Acknowledgments}

A part of this work was promoted in the activity of the AED1099 investigating R\&D committee on techniques for gas-phase simulations of electric discharges and plasmas in the Institute of Electrical Engineers of Japan.

\section{References}

Boyle G J, Tattersall W J, Cocks D G, Dujko S and White R D 2015 Phys. Rev. A 91052710 Christlieb A J, Hitchon W N G, Lawler J E and Lister G G 2009 J. Phys. D: Appl. Phys. 42194007 Date H, Kondo K, Yachi S and Tagashira H 1992 J. Phys. D: Appl. Phys. 25 1330-4

Drallos P J and Wadehra J M 1988 J. Appl. Phys. 63 5601-3

Drallos P J and Wadehra J M 1989 Phys. Rev. A 4 1967-75

Fixel D A and Hitchon W N G 2007 J. Comput. Phys. 227 1387-410

Frost L S and Phelps A V 1962 Phys. Rev. 127 1621-33

Golubovskii Y, Gorchakov S and Uhrlandt D 2013 Plasma Sources Sci. Technol. 22023001 
Golubovskii Y B, Porokhova I A, Lange H, Gortchakov S and Uhrlandt D 2005 Plasma Sources Sci. Technol. 14 45-50

Hitchon W N G, Koch D J and Adams J B 1989 J. Comput. Phys. 83 79-95

Hitchon W N G, Parker G J and Lawler J E 1993 IEEE Trans. Plasma Sci. 21 228-38

Holstein T 1946 Phys. Rev. 70 367-84

Ikuta N and Murakami Y 1987 J. Phys. Soc. Japan 56 115-27

Ikuta N, Takeda A and Yamamoto K 1988 J. Phys. Soc. Japan 57 2401-15

Itoh H, Matsumura T, Satoh K, Date H, Nakao Y and Tagashira H 1993 J. Phys. D: Appl. Phys. 26 $1975-9$

Itoh H, Miura Y, Ikuta N, Nakao Y and Tagashira H 1988 J. Phys. D: Appl. Phys. 21 922-30

Kitamori K, Tagashira H and Sakai Y 1980 J. Phys. D: Appl. Phys. 13 535-50

Kumar K 1995 J. Phys. Soc. Japan 64 4583-8

Kumar K, Skullerud H R and Robson R E 1980 Aust. J. Phys. 33 343-448

Loffhagen D and Winkler R 1996 J. Phys. D: Appl. Phys. 29 618-27

Maeda K and Makabe T 1994 Japan. J. Appl. Phys. 33 4173-6

Makabe T, Nakano N and Yamaguchi Y 1992 Phys. Rev. A 45 2520-31

Mankelevich Y A, Rakhimov A T and Suetin N V 1991 IEEE Trans. Plasma Sci. 19 520-4

Parker G J, Hitchon W N G and Lawler J E 1993 J. Comput. Phys. 106 147-54

Parker J H Jr and Lowke J J 1969 Phys. Rev. 181 290-301

Segur P, Yousfi M and Bordage M C 1984 J. Phys. D: Appl. Phys. 17 2199-214

Segur P, Yousfi M, Kadri M H and Bordage M C 1986 Transport Theory and Stat. Phys. 15 705-57

Shimada T, Nakamura Y, Petrović Z L and Makabe T 2003 J. Phys. D: Appl. Phys. 36 1936-46

Skullerud H R and Kuhn S 1983 J. Phys. D: Appl. Phys. 16 1225-34

Sommerer T J, Hitchon W N G, Harvey R E P and Lawler J E 1991 Phys. Rev. A 43 4452-72

Sommerer T J, Hitchon W N G and Lawler J E 1989 Phys. Rev. A 39 6356-66

Sugawara H 1996 Dissertation, Hokkaido University

Sugawara H and Sakai Y 1999 J. Phys. D: Appl. Phys. 32 1671-80

Sugawara H and Sakai Y 2003 J. Phys. D: Appl. Phys. 36 1994-2000

Sugawara H and Sakai Y 2006 Japan. J. Appl. Phys. 45 5189-96

Sugawara H, Sakai Y and Tagashira H 1992 J. Phys. D: Appl. Phys. 25 1483-7

Sugawara H, Sakai Y and Tagashira H 1994 J. Phys. D: Appl. Phys. 27 90-4

Sugawara H, Sakai Y and Tagashira H 1995 J. Phys. D: Appl. Phys. 28 61-7

Sugawara H, Sakai Y, Tagashira H and Kitamori K 1998 J. Phys. D: Appl. Phys. 31 319-27

Sugawara H, Tagashira H and Sakai Y 1997 J. Phys. D: Appl. Phys. 30 368-73

Tagashira H 1992 Aust. J. Phys. 45 365-74

Tagashira H, Sakai Y and Sakamoto S 1977 J. Phys. D: Appl. Phys. 10 1051-63

Tan W, Hoekstra R J and Kushner Mark J 1996 J. Appl. Phys. 79 3423-31

Thomas W R L 1969 J. Phys. B: At. Mol. Phys. 2 551-61

Wichaidit C and Hitchon W N G 2005 J. Comput. Phys. 203 650-67

Wichaidit C and Hitchon W N G 2005 Phys. Lett. A 335 50-5

Wichaidit C, Hitchon W N G, Lawler J E and Lister G G 2009 J. Phys. D: Appl. Phys. 42025202

Yachi S, Date H, Kitamori K and Tagashira H 1991 J. Phys. D: Appl. Phys. 24 573-80

Yachi S, Kitamura Y, Kitamori K and Tagashira H 1988 J. Phys. D: Appl. Phys. 21 914-21 